\title{
Two Stage Implicit Method on Hexagonal Grids for Approximating the First Derivatives of the Solution to the Heat Equation
}

\author{
Suzan Cival Buranay *(D), Ahmed Hersi Matan and Nouman Arshad
}

check for

updates

Citation: Buranay, S.C.; Matan, A.H.; Arshad N. Two Stage Implicit Method on Hexagonal Grids for Approximating the First Derivatives of the Solution to the Heat Equation. Fractal Fract. 2021, 5, 19. https://doi.org/10.3390/ fractalfract5010019

Academic Editor: Nazim Mahmudov, Rathinasamy Sakthivel and Carlo Cattani

Received: 30 December 2020 Accepted: 20 February 2021 Published: 26 February 2021

Publisher's Note: MDPI stays neutral with regard to jurisdictional claims in published maps and institutional affiliations.

Copyright: (c) 2021 by the authors. Licensee MDPI, Basel, Switzerland. This article is an open access article distributed under the terms and conditions of the Creative Commons Attribution (CC BY) license (https:// creativecommons.org/licenses/by/ $4.0 /)$.
Department of Mathematics, Faculty of Arts and Sciences, Eastern Mediterranean University, Famagusta, North Cyprus, Mersin 10, Turkey; ahmed.hersi@emu.edu.tr (A.H.M.); nouman.arshad@emu.edu.tr (N.A.)

* Correspondence: suzan.buranay@emu.edu.tr

\begin{abstract}
The first type of boundary value problem for the heat equation on a rectangle is considered. We propose a two stage implicit method for the approximation of the first order derivatives of the solution with respect to the spatial variables. To approximate the solution at the first stage, the unconditionally stable two layer implicit method on hexagonal grids given by Buranay and Arshad in 2020 is used which converges with $O\left(h^{2}+\tau^{2}\right)$ of accuracy on the grids. Here, $h$ and $\frac{\sqrt{3}}{2} h$ are the step sizes in space variables $x_{1}$ and $x_{2}$, respectively and $\tau$ is the step size in time. At the second stage, we propose special difference boundary value problems on hexagonal grids for the approximation of first derivatives with respect to spatial variables of which the boundary conditions are defined by using the obtained solution from the first stage. It is proved that the given schemes in the difference problems are unconditionally stable. Further, for $r=\frac{\omega \tau}{h^{2}} \leq \frac{3}{7}$, uniform convergence of the solution of the constructed special difference boundary value problems to the corresponding exact derivatives on hexagonal grids with order $O\left(h^{2}+\tau^{2}\right)$ is shown. Finally, the method is applied on a test problem and the numerical results are presented through tables and figures.
\end{abstract}

Keywords: finite difference method; hexagonal grid; stability analysis; two dimensional heat equation; approximation of derivatives

MSC: 65M06; 65M12; 65M22

\section{Introduction}

Numerical methods have gained considerable attention in many applications, since the exact solution of many problems arising in the models of chemistry, physics, biology, engineering, and many other fields of different sciences is an uphill task. Modeling of these problems leads us to consider a number of physical quantities, representing physical phenomena on a modeling domain. These physical quantities then occur in the model via functions or function derivatives of which for a considerable number of them the Newtonian concept of a derivative satisfies the complexity of the natural occurrences. However, "time's evolution and changes occurring in some systems do not happen in the same manner after a fixed or constant interval of time and do not follow the same routine as one would expect. For instance, a huge variation can occur in a fraction of a second, causing a major change that may affect the whole system's state forever" as stated in [1]. Indeed, it has turned out recently that many of phenomena involved in many branches of chemistry, engineering, biology, ecology, and numerous domains of applied sciences can be described very successfully by models using fractional order differential equations such as acoustic dissipation, viscoelastic systems, mathematical epidemiology, continuous time random walk, and biomedical engineering (see [1] and references therein). Analytical techniques can not solve most of these models with the conventional integer-order derivative, and models with fractional derivatives appearing in practice. Hence, various methods for the solution of these model problems have been developed and proposed in numerous works, in order to provide an improved description of the phenomenon under investigation. 
Common numerical methods include finite difference method, finite element method, finite volume method, variational iteration method, adomian or homotopy analysis, wavelet method, etc.

Most recently, for the model problems of chaotic attractors, exhaustive studies were given for the solvability of chaotic fractional systems with 3D four-scroll attractors in [2], for the Proto-Lorenz system in its chaotic fractional and fractal structure in [3], and for a new auto-replication in systems of attractors with two and three merged basins of attraction via control in [4]. To model some symbiosis systems describing commmensalism and predator-prey processes, the Atangana-Baleanu derivative operator was applied in [5] and a numerical approximation technique was given. Model problems involving a derivative with fractional parameter $\beta$ and the application to transport-convection differential equations were given in [1]. In addition, the use of a control parameter to control on processes related to stationary state system, and on relaxation and diffusion, was studied in [6]. Further, in [7] a comparative analysis between differential fractional operators including the Atangana-Baleanu derivative and the Caputo-Fabrizio derivative applied to the non-linear Kaup-Kupershmidt equation was given and methods of performing numerical approximations of the solutions were presented. Furthermore, for the numerical solution of fractional Volterra type model problems, recent studies include [8] that a class of system of nonlinear singular fractional Volterra integro-differential equations was solved by a proposed computational method. In addition, [9], in which delay-dependent stability switches in fractional differential equations were studied and obtained results were illustrated via a fractional Lotka-Volterra population model. Moreover, [10] as a biological fractional n-species delayed cooperation model of Lotka-Volterra type was presented. Examples to recent studies on numerical solutions of model problems in fractional structure with both stiff and nonstiff components and the leading-edge model problem can be given to [11,12], respectively. A second-order diagonally-implicit-explicit multi-stage integration method was given in [11] for the solution of problems with both stiff and nonstiff components. An implicit method for numerical solution of singular and stiff initial value problem was developed in [12]. For the epidemic models latest studies include [13] that the Crank Nicolson difference scheme and iteration method were used for finding the approximate solution of system of nonlinear observing epidemic model. In addition, [14], in which a novel and time efficient positivity preserving numerical scheme was designed to find the solution of epidemic model involving a reaction-diffusion system in three dimension.

Apart from rectangular grids, hexagonal grids have been also used to develop finite difference methods for the approximate solution of modeled problems in many applied sciences for more than the half century. These studies include the hexagonal grid methods given in meteorological and oceanographic applications by [15-25], of which favorable results were obtained compared with rectangular grids. Hexagonal grids were applied in reservoir simulation in [26] and it was shown that for seven-point floods, hexagonal grid method provides good numerical accuracy at substantially less computational work than rectangular grid method (five or nine point methods). Hexagonal grids were also used in the simulation of electrical wave phenomena propagated in two dimensional reserved-C type cardiac tissue in [27]. The exhibited linear and spiral waves were more efficient than similar computation carried out on rectangular finite volume schemes. Furthermore, hexagonal grids were applied to approximate the solution of the first type boundary value problem of the heat equation in [28-30], convection-diffusion equation in [31], and Dirichlet type boundary value problem of the two dimensional Laplace equation in [32]. In the recent study [29], the solution of first type boundary value problem of heat equation

$$
\frac{\partial u}{\partial t}=\omega\left(\frac{\partial^{2} u}{\partial x_{1}^{2}}+\frac{\partial^{2} u}{\partial x_{2}^{2}}\right)+f\left(x_{1}, x_{2}, t\right),
$$

on special polygons with interior angles $\alpha_{j} \pi, j=1,2, \ldots, M$, for $\alpha_{j} \in\left\{\frac{1}{2}, \frac{1}{3}, \frac{2}{3}\right\}$ where, $\omega>0$ and $f$ is the heat source by using hexagonal grids has been given. Therein, two implicit 
methods named as Difference Problem 1 and Difference Problem 2 both on two layers with 14-points have been proposed. It was assumed that the heat source and the initial and boundary functions are given such that the exact solution belongs to the Hölder space $C_{x, t}^{6+\alpha, 3+\frac{\alpha}{2}}, 0<\alpha<1$. Under this condition, it was proved that the given Difference Problem 1 and Difference Problem 2 converge to the exact solution on the grids with $O\left(h^{2}+\tau^{2}\right)$ and $O\left(h^{4}+\tau\right)$ order of accuracy, respectively.

On the other hand, as well as the solution of the modeled problem, the first order partial derivatives of the solution are also essential to determine the rate of change in the solution and the gradients which determines important phenomena in that model. Such as in the electrostatics the first derivatives of electrostatic potential function define electric field. As the calculation of ray tracing in electrostatic fields by the interpolation methods require the specification at each mesh point not only the potential function $\Phi$ but also the gradients $\left\{\frac{\partial \Phi}{\partial x_{1}}, \frac{\partial \Phi}{\partial x_{2}}\right\}$ and the mixed derivative $\frac{\partial^{2} \Phi}{\partial x_{1} \partial x_{2}}$. Motivated by this aim, in this study a second order accurate two stage implicit method for the approximation of the first order derivatives of the solution $u\left(x_{1}, x_{2}, t\right)$ of (1) with respect to the spatial variables $x_{1}$ and $x_{2}$ on rectangle $D$ is developed.

The research is organized as follows: In Section 2, we consider the first type boundary value problem for the heat equation in (1) on a rectangle $D$ under the assumption that the heat source and the initial and boundary functions are given such that on $\bar{Q}_{T}=\bar{D} \times[0, T]$ the solution $u\left(x_{1}, x_{2}, t\right)$ belongs to the Hölder space $C_{x, t}^{7+\alpha, \frac{7+\alpha}{2}}\left(\bar{Q}_{T}\right), 0<\alpha<1$, where $x=\left(x_{1}, x_{2}\right) \in \bar{D}, t \in[0, T]$, and $\bar{D}$ is the closure of $D$. In addition, hexagonal grid structure and basic notations are given. Further, at the first stage, a two layer implicit method on hexagonal grids given in [29] with $O\left(h^{2}+\tau^{2}\right)$ order of accuracy, where $h$ and $\frac{\sqrt{3}}{2} h$ are the step sizes in space variables $x_{1}$ and $x_{2}$, respectively, and $\tau$ is the step size in time used to approximate the solution $u\left(x_{1}, x_{2}, t\right)$. For the error function when $r \leq \frac{3}{7}$, we provide a pointwise prior estimation depending on $\rho\left(x_{1}, x_{2}, t\right)$, which is the distance from the current grid point to the surface of $Q_{T}$. In Sections 3 and 4, the second stages of the two stage implicit method for the approximation to the first order derivatives of the solution $u\left(x_{1}, x_{2}, t\right)$ with respect to the spatial variables $x_{1}$ and $x_{2}$ are proposed, respectively. It is proved that the constructed implicit schemes at the second stage are unconditionally stable (see Theorem 1 in [33] which gives the sufficient condition of stability). For $r=\frac{\omega \tau}{h^{2}} \leq \frac{3}{7}$, priory error estimations in maximum norm between the exact derivatives $\frac{\partial u}{\partial x_{1}}, \frac{\partial u}{\partial x_{2}}$ and the obtained corresponding approximate solutions are provided giving $O\left(h^{2}+\tau^{2}\right)$ order of accuracy on the hexagonal grids. In Section 5, a numerical example is constructed to support the theoretical results. We applied incomplete block preconditioning given in [34] (see also [35,36]) for the conjugate gradient method to solve the obtained algebraic systems of linear equations for various values of $r$. In Section 6, conclusions and some remarks are given.

\section{First Type Heat Problem and Second Order Accurate Solution on Hexagonal Grids}

Let $D=\left\{\left(x_{1}, x_{2}\right): 0<x_{1}<a_{1}, 0<x_{2}<a_{2}\right\}$ be a rectangle, where $a_{2}$ is multiple of $\sqrt{3}$ and let $\gamma_{j}, j=1,2,3,4$, be the sides of $D$ enumerated in anticlockwise direction starting from the side $x_{1}=0$. Further, $S=\bigcup_{j=1}^{4} \gamma_{j}$ is the boundary of $D$ and denote by $\bar{D}=D \cup S$ the closure of $D$. Let $Q_{T}=D \times(0, T)$, with lateral surface $S_{T}$ more precisely the set of points $(x, t), x=\left(x_{1}, x_{2}\right) \in S$ and $t \in[0, T]$ also $\bar{Q}_{T}$ is the closure of $Q_{T}$. We consider the first type boundary value problem for the two space dimensional heat equation: 


\section{BVP(1):}

$$
\begin{aligned}
\frac{\partial u}{\partial t} & =\omega\left(\frac{\partial^{2} u}{\partial x_{1}^{2}}+\frac{\partial^{2} u}{\partial x_{2}^{2}}\right)+f\left(x_{1}, x_{2}, t\right) \text { on } Q_{T}, \\
u\left(x_{1}, x_{2}, 0\right) & =\varphi\left(x_{1}, x_{2}\right) \text { on } \bar{D} \\
u\left(x_{1}, x_{2}, t\right) & =\phi\left(x_{1}, x_{2}, t\right) \text { on } S_{T},
\end{aligned}
$$

where $\omega$ is a positive constant. We assume that the heat source function $f\left(x_{1}, x_{2}, t\right)$ and the initial and boundary functions $\varphi\left(x_{1}, x_{2}\right)$ and $\phi\left(x_{1}, x_{2}, t\right)$, respectively, are given such that the Problems (2)-(4) has a unique solution $u$ belonging to the Hölder class $C_{x, t}^{7+\alpha, \frac{7+\alpha}{2}}\left(\bar{Q}_{T}\right)$. For the smoothness of solutions of parabolic equations in regions with edges, see [37] for the Dirichlet and [38] for the mixed boundary value problems. Let $h>0$, with $h=a_{1} / N_{1}$, where $N_{1}$ is positive integer and assign $D^{h}$ a hexagonal grid on $D$, with step size $h$, defined as the set of nodes

$$
\begin{aligned}
D^{h}= & \left\{x=\left(x_{1}, x_{2}\right) \in D: x_{1}=\frac{i^{\prime}-j^{\prime}}{2} h, x_{2}=\frac{\sqrt{3}\left(i^{\prime}+j^{\prime}\right)}{2} h,\right. \\
& \left.i^{\prime}=1,2, \ldots ; j^{\prime}=0 \pm 1 \pm 2, \ldots\right\} .
\end{aligned}
$$

Let $\gamma_{j}^{h}, j=1, \ldots, 4$ be the set of nodes on the interior of $\gamma_{j}$ and let $\widehat{\gamma}_{j}^{h}=\gamma_{j-1} \cap \gamma_{j}$ be the $j$ th vertex of $D, S^{h}=\bigcup_{j=1}^{4}\left(\gamma_{j}^{h} \cup \widehat{\gamma}_{j}^{h}\right), \bar{D}^{h}=D^{h} \cup S^{h}$. Further, let $D^{* l h}, D^{* r h}$ denote the set of interior nodes whose distance from the boundary is $\frac{h}{2}$ and the hexagon has a left ghost point as shown in Figure 1 or a right ghost point as presented in Figure 2, emerging through the left or right side of the rectangle, respectively. We also denote by $D^{* h}=D^{* l h} \cup D^{* r h}$ and $D^{0 h}=D^{h} \backslash D^{* h}$.

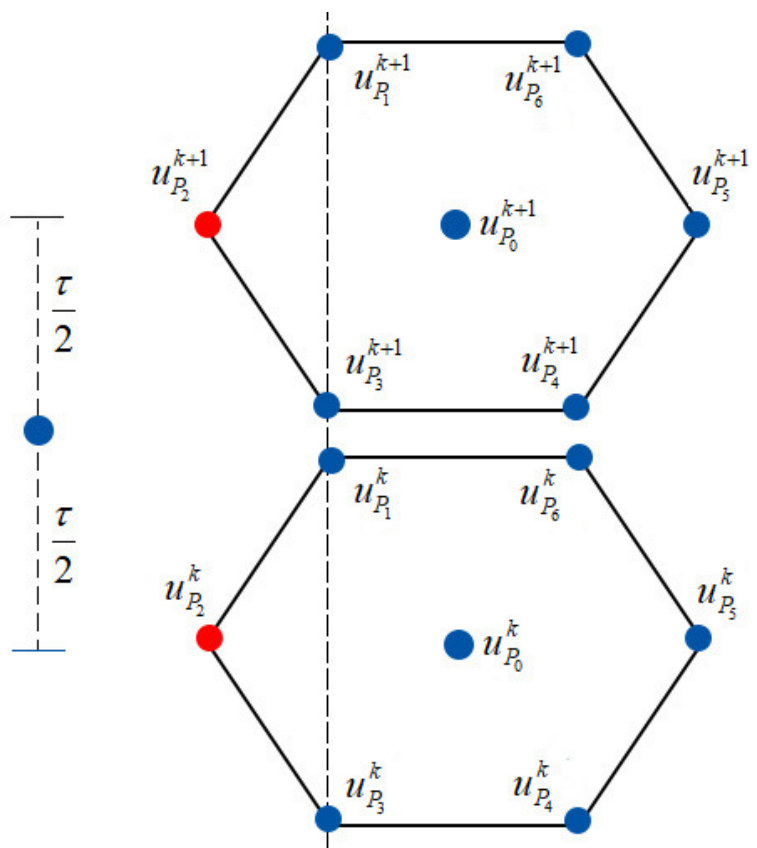

Figure 1. The solution $u_{P_{2}}^{k}$ and $u_{P_{2}}^{k+1}$ on the left ghost points at time moments $t=k \tau$ and $(k+1) \tau$, respectively. 


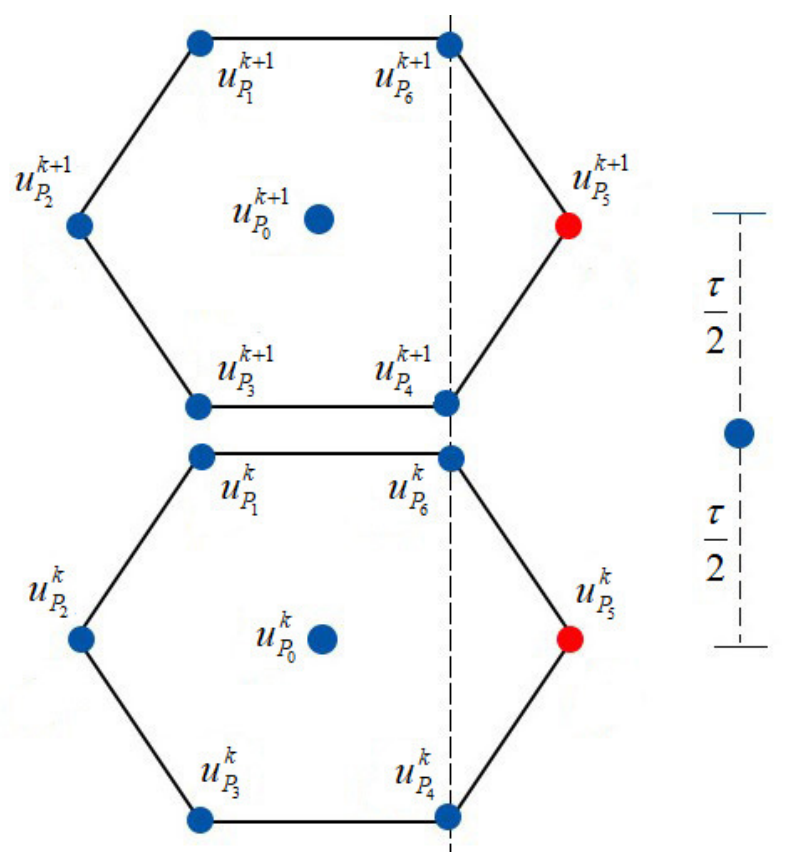

Figure 2. The solution $u_{P_{5}}^{k}$ and $u_{P_{5}}^{k+1}$ on the right ghost points at time moments $t=k \tau$ and $(k+1) \tau$, respectively.

Next, let

$$
\begin{aligned}
& \gamma_{\tau}=\left\{t_{k}=k \tau, \tau=\frac{T}{M^{\prime}}, k=1, \ldots, M^{\prime}\right\} \\
& \bar{\gamma}_{\tau}=\left\{t_{k}=k \tau, \tau=\frac{T}{M^{\prime}}, k=0, \ldots, M^{\prime}\right\},
\end{aligned}
$$

and the set of internal nodes and lateral surface nodes be defined by

$$
\begin{aligned}
D^{h} \gamma_{\tau} & =D^{h} \times \gamma_{\tau}=\left\{(x, t): x=\left(x_{1}, x_{2}\right) \in D^{h}, t \in \gamma_{\tau}\right\}, \\
S_{T}^{h} & =S^{h} \times \bar{\gamma}_{\tau}=\left\{(x, t): x=\left(x_{1}, x_{2}\right) \in S^{h}, t \in \bar{\gamma}_{\tau}\right\},
\end{aligned}
$$

respectively. Let $D^{* l h} \gamma_{\tau}=D^{* l h} \times \gamma_{\tau} \subset D^{h} \gamma_{\tau}$ and $D^{* r h} \gamma_{\tau}=D^{* r h} \times \gamma_{\tau} \subset D^{h} \gamma_{\tau}$ and $D^{* h} \gamma_{\tau}=D^{* l h} \gamma_{\tau} \cup D^{* r h} \gamma_{\tau}$. In addition, $D^{0 h} \gamma_{\tau}=D^{h} \gamma_{\tau} \backslash D^{* h} \gamma_{\tau}$ and $\overline{D^{h} \gamma_{\tau}}$ is the closure of $D^{h} \gamma_{\tau}$

Let $P_{0}$ denote the center of the hexagon and $P a t t\left(P_{0}\right)$ denote the pattern of the hexagon consisting the neighboring points $P_{i}, i=1, \ldots, 6$. In addition, $u_{P_{i}}^{k+1}$ denotes the exact solution at the point $P_{i}$ and $u_{P_{A}}^{k+1}$ denotes the value at the boundary point for the time moment $t+\tau$ as follows:

$$
\begin{aligned}
& u_{P_{1}}^{k+1}=u\left(x_{1}-\frac{h}{2}, x_{2}+\frac{\sqrt{3}}{2} h, t+\tau\right), u_{P_{3}}^{k+1}=u\left(x_{1}-\frac{h}{2}, x_{2}-\frac{\sqrt{3}}{2} h, t+\tau\right), \\
& u_{P_{2}}^{k+1}=u\left(x_{1}-h, x_{2}, t+\tau\right), u_{P_{5}}^{k+1}=u\left(x_{1}+h, x_{2}, t+\tau\right), \\
& u_{P_{4}}^{k+1}=u\left(x_{1}+\frac{h}{2}, x_{2}-\frac{\sqrt{3}}{2} h, t+\tau\right), u_{P_{6}}^{k+1}=u\left(x_{1}+\frac{h}{2}, x_{2}+\frac{\sqrt{3}}{2} h, t+\tau\right), \\
& u_{P_{0}}^{k+1}=u\left(x_{1}, x_{2}, t+\tau\right), u_{P_{A}}^{k+1}=u\left(\hat{p}, x_{2}, t+\tau\right),\left(\hat{p}, x_{2}, t+\tau\right) \in S_{T}^{h},
\end{aligned}
$$

where the value of $\hat{p}=0$ if $P_{0} \in D^{* l h} \gamma_{\tau}$ and $\hat{p}=a_{1}$ if $P_{0} \in D^{* r h} \gamma_{\tau}$ as also given in (21). Analogously, the values $u_{P_{i}}^{k}, i=0, \ldots, 6$ and $u_{P_{A}}^{k}$ present the exact solution at the same space coordinates of $P_{i}, i=0, \ldots, 6$ and $P_{A}$, respectively, but at time level $t=k \tau$. Further, $u_{h, \tau, P_{i}{ }^{\prime}}$, 
$i=0, \ldots, 6, u_{h, \tau, P_{A}}^{k+1}$ and $u_{h, \tau, P_{i}}^{k}, i=0, \ldots, 6, u_{h, \tau, P_{A}}^{k}$ present the numerical solution at the same space coordinates of $P_{i}, i=0, \ldots, 6$ and $P_{A}$ for time moments $t+\tau$ and $t=k \tau$, respectively and $f_{P_{0}}^{k+\frac{1}{2}}=f\left(x_{1}, x_{2}, t+\frac{\tau}{2}\right)$, and $f_{P_{A}}^{k+1}=f\left(\widehat{p}, x_{2}, t+\tau\right)$. For the numerical solution of the BVP(1) the following difference problem (named as Difference Problem 1) was given in [29] which we will consider as the Stage $1\left(H^{2 n d}\right)$ of the two stage implicit method:

$$
\begin{aligned}
& \text { Stage 1 }\left(H^{2 n d}\right) \\
& \qquad \begin{aligned}
\Theta_{h, \tau} u_{h, \tau}^{k+1} & =\Lambda_{h, \tau} u_{h, \tau}^{k}+\psi \text { on } D^{0 h} \gamma_{\tau}, \\
\Theta_{h, \tau}^{*} u_{h, \tau}^{k+1} & =\Lambda_{h, \tau}^{*} u_{h, \tau}^{k}+\Gamma_{h, \tau}^{*} \phi+\psi^{*} \text { on } D^{* h} \gamma_{\tau}, \\
u_{h, \tau} & =\varphi\left(x_{1}, x_{2}\right), t=0 \text { on } \bar{D}^{h}, \\
u_{h, \tau} & =\phi\left(x_{1}, x_{2}, t\right) \text { on } S_{T}^{h},
\end{aligned}
\end{aligned}
$$

for $k=0, \ldots, M^{\prime}-1$, where

$$
\begin{aligned}
& \psi=f_{P_{0}}^{k+\frac{1}{2}}, \\
& \psi^{*}=f_{P_{0}}^{k+\frac{1}{2}}-\frac{1}{6} f_{P_{A}}^{k+\frac{1}{2}}, \\
& \Theta_{h, \tau} u^{k+1}=\left(\frac{1}{\tau}+\frac{2 \omega}{h^{2}}\right) u_{P_{0}}^{k+1}-\frac{\omega}{3 h^{2}} \sum_{i=1}^{6} u_{P_{i}}^{k+1}, \\
& \Lambda_{h, \tau} u^{k}=\left(\frac{1}{\tau}-\frac{2 \omega}{h^{2}}\right) u_{P_{0}}^{k}+\frac{\omega}{3 h^{2}} \sum_{i=1}^{6} u_{P_{i}}^{k} \\
& \Theta_{h, \tau}^{*} u^{k+1}=\left(\frac{1}{\tau}+\frac{7 \omega}{3 h^{2}}\right) u_{P_{0}}^{k+1}-\frac{\omega}{3 h^{2}}\left(u\left(p+\eta, x_{2}, t+\tau\right)\right. \\
& \left.+u\left(p, x_{2}+\frac{\sqrt{3}}{2} h, t+\tau\right)+u\left(p, x_{2}-\frac{\sqrt{3}}{2} h, t+\tau\right)\right), \\
& \Lambda_{h, \tau}^{*} u^{k}=\left(\frac{1}{\tau}-\frac{7 \omega}{3 h^{2}}\right) u_{P_{0}}^{k}+\frac{\omega}{3 h^{2}}\left(u\left(p, x_{2}+\frac{\sqrt{3}}{2} h, t\right)\right. \\
& \left.+u\left(p, x_{2}-\frac{\sqrt{3}}{2} h, t\right)+u\left(p+\eta, x_{2}, t\right)\right), \\
& \Gamma_{h, \tau}^{*} \phi=\frac{2 \omega}{9 h^{2}}\left(\phi\left(\widehat{p}, x_{2}+\frac{\sqrt{3}}{2} h, t+\tau\right)+\phi\left(\widehat{p}, x_{2}-\frac{\sqrt{3}}{2} h, t+\tau\right)\right. \\
& \left.+\phi\left(\widehat{p}, x_{2}+\frac{\sqrt{3}}{2} h, t\right)+\phi\left(\widehat{p}, x_{2}-\frac{\sqrt{3}}{2} h, t\right)\right) \\
& +\left(\frac{1}{6 \tau}+\frac{8 \omega}{9 h^{2}}\right) \phi\left(\widehat{p}, x_{2}, t+\tau\right)+\left(-\frac{1}{6 \tau}+\frac{8 \omega}{9 h^{2}}\right) \phi\left(\widehat{p}, x_{2}, t\right),
\end{aligned}
$$

and

$$
\left\{\begin{array}{c}
p=h, \widehat{p}=0, \eta=\frac{h}{2} \text { if } P_{0} \in D^{* l h} \gamma_{\tau} \\
p=a_{1}-h, \widehat{p}=a_{1}, \eta=-\frac{h}{2} \text { if } P_{0} \in D^{* h} \gamma_{\tau}
\end{array}\right.
$$


By numbering the interior grid points using standard ordering as $L_{j}, j=1,2, \ldots, N$, the obtained algebraic linear system of equations in matrix form given in [29] is as follows:

$$
A \widetilde{u}^{k+1}=B \widetilde{u}^{k}+\tau q_{u}^{k}
$$

where $A, B \in R^{N \times N}$ are

$$
A=\left(I+\frac{\omega \tau}{h^{2}} C\right), B=\left(I-\frac{\omega \tau}{h^{2}} C\right),
$$

and

$$
C=D_{1}-\frac{1}{3} I n c \in R^{N \times N},
$$

and $\widetilde{u}^{k}, q_{u}^{k} \in R^{N}$. The matrix Inc is the incidence matrix of the neighboring topology with entries unity for the points in the pattern of the hexagon center. In addition, $I$ is the identity matrix, $D_{1}$ is a diagonal matrix with entries

$$
d_{1, j j}=\left\{\begin{array}{c}
2 \text { if } L_{j} \in D^{0 h} \gamma_{\tau} \\
\frac{7}{3} \text { if } L_{j} \in D^{* h} \gamma_{\tau}
\end{array}, j=1,2, \ldots, N\right.
$$

Lemma 1. (a) The matrix $A$ in (22) is a nonsingular M-matrix and is also a symmetric positive definite matrix.

(b) $\left\|A^{-1}\right\|_{2}<1$ and $\left\|A^{-1} B\right\|_{2}<1$ for $r=\frac{\omega \tau}{h^{2}}>0$.

Proof. Proof is given in ([29]).

Let

$$
\varepsilon_{h, \tau}^{u}=u_{h, \tau}-u \text { on } \overline{D^{h} \gamma_{\tau}} .
$$
in [29]

From (10)-(13) and (26), the error function $\varepsilon_{h, \tau}^{u}$ satisfies the following system as given

$$
\begin{aligned}
\Theta_{h, \tau} \varepsilon_{h, \tau}^{u, k+1} & =\Lambda_{h, \tau} \varepsilon_{h, \tau}^{u, k}+\Psi_{1}^{u, k} \text { on } D^{0 h} \gamma_{\tau}, \\
\Theta_{h, \tau}^{*} \varepsilon_{h, \tau}^{u, k+1} & =\Lambda_{h, \tau}^{*} \varepsilon_{h, \tau}^{u, k}+\Psi_{2}^{u, k} \text { on } D^{* h} \gamma_{\tau}, \\
\varepsilon_{h, \tau}^{u} & =0, t=0 \text { on } \bar{D}^{h}, \\
\varepsilon_{h, \tau}^{u} & =0 \text { on } S_{T}^{h},
\end{aligned}
$$

where

$$
\begin{aligned}
& \Psi_{1}^{u, k}=\Lambda_{h, \tau} u^{k}-\Theta_{h, \tau} u^{k+1}+\psi, \\
& \Psi_{2}^{u, k}=\Lambda_{h, \tau}^{*} u^{k}-\Theta_{h, \tau}^{*} u^{k+1}+\Gamma_{h, \tau}^{*} \phi+\psi^{*},
\end{aligned}
$$

and $\psi, \psi^{*}$, and $\phi$ are the given functions in (10), (11), and (13), respectively.

Pointwise Priory Estimation For the Error Function (27)-(30)

Consider the following systems

$$
\begin{aligned}
\Theta_{h, \tau} \widehat{\vartheta}_{h, \tau}^{k+1} & =\Lambda_{h, \tau} \widehat{q}_{h, \tau}^{k}+\widehat{g}_{1}^{k} \text { on } D^{0 h} \gamma_{\tau}, \\
\Theta_{h, \tau}^{*} \widehat{q}_{h, \tau}^{k+1} & =\Lambda_{h, \tau}^{*} \widehat{q}_{h, \tau}^{k}+\Gamma_{h, \tau}^{*} \widehat{q}_{\phi, h, \tau}+\widehat{g}_{2}^{k} \text { on } D^{* h} \gamma_{\tau}, \\
\widehat{q}_{h, \tau} & =\widehat{q}_{\phi, h, \tau}, t=0 \text { on } \bar{D}^{h}, \\
\widehat{q}_{h, \tau} & =\widehat{q}_{\phi, h, \tau} \text { on } S_{T}^{h},
\end{aligned}
$$




$$
\begin{aligned}
\Theta_{h, \tau} \bar{q}_{h, \tau}^{k+1} & =\Lambda_{h, \tau} \bar{q}_{h, \tau}^{k}+\bar{g}_{1}^{k} \text { on } D^{0 h} \gamma_{\tau}, \\
\Theta_{h, \tau}^{*} \bar{q}_{h, \tau}^{+1} & =\Lambda_{h, \tau}^{*} \bar{q}_{h, \tau}^{k}+\Gamma_{h, \tau}^{*} \bar{q}_{\phi, h, \tau}+\bar{g}_{2}^{k} \text { on } D^{* h} \gamma_{\tau}, \\
\bar{q}_{h, \tau} & =\bar{q}_{\phi, h, \tau}, t=0 \text { on } \bar{D}^{h}, \\
\bar{q}_{h, \tau} & =\bar{q}_{\phi, h, \tau} \text { on } S_{T}^{h},
\end{aligned}
$$

for $k=0, \ldots, M^{\prime}-1$, where $\widehat{g}_{1}, \widehat{g}_{2}$ and $\bar{g}_{1}, \bar{g}_{2}$ are given functions. The algebraic systems (33)-(36) and (37)-(40) can be written in matrix form

$$
\begin{aligned}
& A \widehat{q}^{k+1}=B \widehat{q}^{k}+\tau \widehat{g}^{k}, \\
& A \bar{q}^{k+1}=B \bar{q}^{k}+\tau \bar{g}^{k},
\end{aligned}
$$

respectively, for every time level $k=0, \ldots, M^{\prime}-1$ where $A$ and $B$ are the matrices given in (22) and $\widehat{q}^{k}, \bar{q}^{k}, \widehat{g}^{k}, \bar{g}^{k} \in R^{N}$. We also use the partial order $K_{1} \leq K_{2}$ which means that $K_{1}-K_{2} \leq 0$ is nonpositive and $K_{1} \geq K_{2}$ means that $K_{1}-K_{2} \geq 0$ is nonnegative wherever they present matrices in $R^{N \times N}$ or vectors in $R^{N}$.

Lemma 2. Let $\widehat{q}^{k+1}$ be the solution of the difference Equation (41) and $\bar{q}^{k+1}$ be the solution of the difference Equation (42). For $r=\frac{\omega \tau}{h^{2}} \leq \frac{3}{7}$, if

$$
\begin{aligned}
\bar{q}^{0} & \geq 0 \text { and } \bar{g}^{k} \geq 0, \\
\left|\widehat{q}^{0}\right| & \leq \bar{q}^{0}, \\
\left|\widehat{g}^{k}\right| & \leq \bar{g}^{k},
\end{aligned}
$$

for $k=0, \ldots, M^{\prime}-1$, then

$$
\bar{q}^{k+1} \geq 0 \text { and }\left|\widehat{q}^{k+1}\right| \leq \bar{q}^{k+1} \text { for } k=0, \ldots, M^{\prime}-1 .
$$

Proof. On the basis of Lemma $1, A^{-1} \geq 0$ and if $r=\frac{\omega \tau}{h^{2}} \leq \frac{3}{7}$ then $B \geq 0$ and from (43) we have $\bar{g}^{k} \geq 0, k=0, \ldots, M^{\prime}-1$ and $\bar{q}^{0} \geq 0$. Then, assume that $\bar{q}^{k} \geq 0$ by using induction we have

$$
\bar{q}^{k+1}=A^{-1} B \bar{q}^{k}+\tau A^{-1} \bar{g}^{k} \geq 0,
$$

which gives $\bar{q}^{k+1} \geq 0, k=0, \ldots, M^{\prime}-1$. In addition, $\left|\widehat{q}^{0}\right| \leq \bar{q}^{0}$ from (44). Next assume that $\left|\widehat{q}^{k}\right| \leq \bar{q}^{k}$, by using (45) and induction gives

$$
\begin{aligned}
\hat{q}^{k+1} & =A^{-1} B \widehat{q}^{k}+\tau A^{-1} \widehat{g}^{k} \\
\left|\widehat{q}^{k+1}\right| & \leq A^{-1} B\left|\widehat{q}^{k}\right|+\tau A^{-1}\left|\widehat{g}^{k}\right| \\
& \leq A^{-1} B \bar{q}^{k}+\tau A^{-1} \bar{g}^{k}=\bar{q}^{k+1} .
\end{aligned}
$$

Thus, we obtain (46).

Let

$$
\begin{aligned}
& S_{T \gamma_{1}}=\gamma_{1} \times(0, T]=\left\{\left(0, x_{2}, t\right):\left(0, x_{2}\right) \in \gamma_{1}, t \in(0, T]\right\}, \\
& S_{T} \gamma_{2}=\gamma_{2} \times(0, T]=\left\{\left(x_{1}, 0, t\right):\left(x_{1}, 0\right) \in \gamma_{2}, t \in(0, T]\right\}, \\
& S_{T} \gamma_{3}=\gamma_{3} \times(0, T]=\left\{\left(a_{1}, x_{2}, t\right):\left(a_{1}, x_{2}\right) \in \gamma_{3}, t \in(0, T]\right\} \\
& S_{T} \gamma_{4}=\gamma_{4} \times(0, T]=\left\{\left(x_{1}, a_{2}, t\right):\left(x_{1}, a_{2}\right) \in \gamma_{4}, t \in(0, T]\right\} \\
& S_{T} \gamma_{5}=\left\{\left(x_{1}, x_{2}, 0\right):\left(x_{1}, x_{2}\right) \in \bar{D}, t=0\right\}
\end{aligned}
$$


and $S_{T}^{h} \gamma_{i}, i=1,2, \ldots, 5$ denote the corresponding sets of grid points. In addition, let $F=\bigcup_{i=1}^{5} S_{T} \gamma_{i}$ denote the surface of $Q_{T}$.

Theorem 1. For the solution of the problem (27)-(30), the following inequality holds true

$$
\left|\varepsilon_{h, \tau}^{u}\right| \leq d \Omega_{1}(h, \tau) \rho\left(x_{1}, x_{2}, t\right) \text {, on } \overline{D^{h} \gamma_{\tau}},
$$

for $r=\frac{\omega \tau}{h^{2}} \leq \frac{3}{7}$ where

$$
\begin{gathered}
\Omega_{1}(h, \tau)=\frac{1}{24} \tau^{2}(1+6 \omega) \beta^{*}+\frac{3 \omega}{10} h^{2} \alpha^{*}, \\
\alpha^{*}=\max \left\{\max _{\bar{Q}_{T}}\left|\frac{\partial^{4} u}{\partial x_{1}^{4}}\right|, \overline{m a x}_{\bar{Q}_{T}}\left|\frac{\partial^{4} u}{\partial x_{2}^{4}}\right|, \max _{\bar{Q}_{T}}\left|\frac{\partial^{4} u}{\partial x_{1}^{2} \partial x_{2}^{2}}\right|\right\}, \\
\beta^{*}=\max \left\{\max _{\overline{\mathrm{Q}}_{T}}\left|\frac{\partial^{3} u}{\partial t^{3}}\right|, \max _{\overline{\mathrm{Q}}_{T}}\left|\frac{\partial^{4} u}{\partial x_{2}^{2} \partial t^{2}}\right|, \max _{\overline{\mathrm{Q}}_{T}}\left|\frac{\partial^{4} u}{\partial x_{1}^{2} \partial t^{2}}\right|\right\}, \\
d=\max \left\{\frac{a_{1}}{2 \omega}, \frac{a_{2}}{2 \omega^{\prime}}, 1\right\},
\end{gathered}
$$

and $u$ is the exact solution of $\boldsymbol{B V P}(\mathbf{1})$ and $\rho\left(x_{1}, x_{2}, t\right)$ is the distance from the current grid point in $\overline{D^{h} \gamma_{\tau}}$ to the surface $F$ of $Q_{T}$.

Proof. We consider the system

$$
\begin{aligned}
\Theta_{h, \tau} \widehat{\varepsilon}_{h, \tau}^{u, k+1} & =\Lambda_{h, \tau} \widehat{\varepsilon}_{h, \tau}^{u, k}+\Omega_{1}(h, \tau) \text { on } D^{0 h} \gamma_{\tau}, \\
\Theta_{h, \tau}^{*} \widehat{\varepsilon}_{h, \tau}^{u, k+1} & =\Lambda_{h, \tau}^{*} \widehat{\varepsilon}_{h, \tau}^{u, k}+\frac{5}{6} \Omega_{1}(h, \tau) \text { on } D^{* h} \gamma_{\tau} \\
\widehat{\varepsilon}_{h, \tau}^{u} & =\widehat{\varepsilon}_{\varphi, h, \tau}^{u}=0, t=0 \text { on } \bar{D}^{h}, \\
\widehat{\varepsilon}_{h, \tau}^{u} & =\widehat{\varepsilon}_{\phi, h, \tau}^{u}=0 \text { on } S_{T}^{h},
\end{aligned}
$$

and the majorant functions

$$
\begin{aligned}
& \bar{\varepsilon}_{1}^{u}\left(x_{1}, x_{2}, t\right)=\frac{1}{2 \omega} \Omega_{1}(h, \tau)\left(a_{1} x_{1}-x_{1}^{2}\right) \geq 0 \text { on } \overline{D^{h} \gamma_{\tau}}, \\
& \bar{\varepsilon}_{2}^{u}\left(x_{1}, x_{2}, t\right)=\frac{1}{2 \omega} \Omega_{1}(h, \tau)\left(a_{2} x_{2}-x_{2}^{2}\right) \geq 0 \text { on } \overline{D^{h} \gamma_{\tau}}, \\
& \bar{\varepsilon}_{3}^{u}\left(x_{1}, x_{2}, t\right)=\Omega_{1}(h, \tau) t \geq 0 \text { on } \overline{D^{h} \gamma_{\tau}}
\end{aligned}
$$

in which $\bar{\varepsilon}_{i}^{u}, i=1,2,3$ satisfy the difference boundary value problem

$$
\begin{aligned}
\Theta_{h, \tau} \bar{\varepsilon}_{i, h, \tau}^{u, k+1} & =\Lambda_{h, \tau} \bar{\varepsilon}_{i, h, \tau}^{u, k}+\Omega_{1}(h, \tau) \text { on } D^{0 h} \gamma_{\tau}, \\
\Theta_{h, \tau}^{*} \bar{\varepsilon}_{i, h, \tau}^{u, k+1} & =\Lambda_{h, \tau}^{*} \bar{\varepsilon}_{i, h, \tau}^{u, k}+\Gamma_{h, \tau}^{*} \bar{\varepsilon}_{i, \phi, h, \tau}^{u}+\frac{5}{6} \Omega_{1}(h, \tau) \text { on } D^{* h} \gamma_{\tau}, \\
\bar{\varepsilon}_{i, h, \tau}^{u} & =\bar{\varepsilon}_{i, \varphi, h, \tau}^{u}=\bar{\varepsilon}_{i}^{u}\left(x_{1}, x_{2}, 0\right) \geq 0, t=0 \text { on } \bar{D}^{h}, \\
\bar{\varepsilon}_{i, h, \tau}^{u} & =\bar{\varepsilon}_{i, \phi, h, \tau}^{u} \geq 0 \text { on } S_{T}^{h} .
\end{aligned}
$$


Therefore, we write the difference problems (56)-(59) and (63)-(66) for fixed $k \geq 0$ in matrix form

$$
\begin{aligned}
& A \widehat{\varepsilon}^{u, k+1}=B \widehat{\varepsilon}^{u, k}+\tau \widehat{e}^{u, k}, \\
& A \bar{\varepsilon}_{i}^{u, k+1}=B \bar{\varepsilon}_{i}^{u, k}+\tau \bar{e}_{i}^{u, k}, i=1,2,3,
\end{aligned}
$$

respectively, where $A$ and $B$ are the matrices given in (22) and $\bar{e}_{i}^{u, k}, \bar{\varepsilon}_{i}^{u, k}, i=1,2,3$ and $\widehat{\varepsilon}^{u, k}, \hat{e}^{u, k} \in R^{N}$. Using (52) and (56)-(66) gives $\bar{\varepsilon}_{i}^{u, 0} \geq 0,\left|\widehat{\varepsilon}^{u, 0}\right| \leq \bar{\varepsilon}_{i}^{u, 0}$, and $\bar{e}_{i}^{u, k} \geq 0$, and $\left|\widehat{e}^{u, k}\right| \leq \bar{e}_{i}^{u, k}, i=1,2,3$, for $k=0, \ldots, M^{\prime}-1$. On the basis of Lemma 2, we get $\left|\widehat{\varepsilon}^{u, k+1}\right| \leq$ $\bar{\varepsilon}_{i}^{u, k+1}, k=0, \ldots, M^{\prime}-1$ and using that $\Omega_{1}(h, \tau) \geq\left|\Psi_{1}^{u, k}\right|$ on $D^{0 h} \gamma_{\tau}$, and $\frac{5}{6} \Omega_{1}(h, \tau) \geq\left|\Psi_{2}^{u, k}\right|$ on $D^{* h} \gamma_{\tau}$ gives

$$
\left|\varepsilon_{h, \tau}^{u}\right| \leq \min _{i=1,2,3} \bar{\varepsilon}_{i}^{u}\left(x_{1}, x_{2}, t\right) \leq d \Omega_{1}(h, \tau) \rho\left(x_{1}, x_{2}, t\right) \text { on } \overline{D^{h} \gamma_{\tau}},
$$

\section{Difference Problem Approximating $\frac{\partial u}{\partial x_{1}}$ on Hexagonal grids with $O\left(h^{2}+\tau^{2}\right)$ Order of Accuracy}

We use the notations $\partial_{x_{1}} f_{P_{0}}^{k+\frac{1}{2}}=\left.\frac{\partial f}{\partial x_{1}}\right|_{\left(x_{1}, x_{2}, t+\frac{\tau}{2}\right)}$ and $\partial_{x_{1}} f_{P_{A}}^{k+\frac{1}{2}}=\left.\frac{\partial f}{\partial x_{1}}\right|_{\left(\hat{p}, x_{2}, t+\frac{\tau}{2}\right)}$. Given the boundary value Problems (2)-(4), we denote $p_{i}=\frac{\partial u}{\partial x_{1}}$ on $S_{T} \gamma_{i}, i=1,2, \ldots, 5$ and setup the next boundary value problem for $v=\frac{\partial u}{\partial x_{1}}$.

BVP(2):

$$
\begin{aligned}
L v & =\frac{\partial f\left(x_{1}, x_{2}, t\right)}{\partial x_{1}} \text { on } Q_{T} \\
v\left(x_{1}, x_{2}, t\right) & =p_{i} \text { on } S_{T} \gamma_{i}, i=1,2, \ldots, 5
\end{aligned}
$$

where

$$
L \equiv \frac{\partial}{\partial t}-\omega\left(\frac{\partial^{2}}{\partial x_{1}^{2}}+\frac{\partial^{2}}{\partial x_{2}^{2}}\right),
$$

and $f\left(x_{1}, x_{2}, t\right)$ is the given function in (2). On the basis of the assumption that $u \in$ $C_{x, t}^{7+\alpha, \frac{7+\alpha}{2}}\left(\bar{Q}_{T}\right)$, we assume that the solution $v \in C_{x, t}^{6+\alpha, 3+\frac{\alpha}{2}}\left(\bar{Q}_{T}\right)$.

We take

$$
\begin{aligned}
p_{1 h}^{2^{n d}}= & \left\{\begin{array}{c}
\frac{1}{2 h}\left(-3 u\left(0, x_{2}, t\right)+4 u_{h, \tau}\left(h, x_{2}, t\right)\right. \\
\left.-u_{h, \tau}\left(2 h, x_{2}, t\right)\right) \text { if } P_{0} \in D^{0 h} \gamma_{\tau} \\
\frac{1}{3 h}\left(-8 u\left(0, x_{2}, t\right)+9 u_{h, \tau}\left(\frac{h}{2}, x_{2}, t\right) \text { on } S_{T}^{h} \gamma_{1},\right. \\
\left.-u_{h, \tau}\left(\frac{3 h}{2}, x_{2}, t\right)\right) \text { if } P_{0} \in D^{* l h} \gamma_{\tau}
\end{array}\right. \\
p_{3 h}^{2^{n d}}= & \left\{\begin{array}{c}
\frac{1}{2 h}\left(3 u\left(a_{1}, x_{2}, t\right)-4 u_{h, \tau}\left(a_{1}-h, x_{2}, t\right)\right. \\
\left.+u_{h, \tau}\left(a_{1}-2 h, x_{2}, t\right)\right) \text { if } P_{0} \in D^{0 h} \gamma_{\tau} \\
\frac{1}{3 h}\left(8 u\left(a_{1}, x_{2}, t\right)-9 u_{h, \tau}\left(a_{1}-\frac{h}{2}, x_{2}, t\right) \text { on } S_{T}^{h} \gamma_{3},\right. \\
\left.+u_{h, \tau}\left(a_{1}-\frac{3 h}{2}, x_{2}, t\right)\right) \text { if } P_{0} \in D^{* r h} \gamma_{\tau}
\end{array}\right. \\
p_{i h}= & \frac{\partial \phi\left(x_{1}, x_{2}, t\right)}{\partial x_{1}} \text { on } S_{T}^{h} \gamma_{i}, i=2,4 \\
p_{5 h}= & \frac{\partial \varphi\left(x_{1}, x_{2}\right)}{\partial x_{1}} \text { on } S_{T}^{h} \gamma_{5},
\end{aligned}
$$

and $\varphi\left(x_{1}, x_{2}\right)$ given in (3), $\phi\left(x_{1}, x_{2}, t\right)$ given in (4) are the initial and boundary functions, respectively, $u_{h, \tau}$ is the solution of the difference problem in Stage $\mathbf{1}\left(H^{2 n d}\right)$. 
Lemma 3. The following inequality

$$
\left|p_{i h}^{2^{\text {nd }}}\left(u_{h, \tau}\right)-p_{i h}^{2^{n d}}(u)\right| \leq 3 d \Omega_{1}(h, \tau), \quad i=1,3 .
$$

holds true for $r=\frac{\omega \tau}{h^{2}} \leq \frac{3}{7}$, where $u$ is the solution of the boundary value Problems (2)-(4) and $u_{h, \tau}$ is the solution of the difference problem in Stage $1\left(H^{2 n d}\right)$ and $\Omega_{1}(h, \tau)$ and $d$ are as given in (52) and (55), respectively.

Proof. Taking into consideration Theorem 1, and using (51), (73), and (74) when $P_{0} \in$ $D^{0 h} \gamma_{\tau}$, we have

$$
\begin{aligned}
\left|p_{i h}^{2^{n d}}\left(u_{h, \tau}\right)-p_{i h}^{2^{n d}}(u)\right| & \leq \frac{1}{2 h}\left(4 h d \Omega_{1}(h, \tau)+2 h d \Omega_{1}(h, \tau)\right) \\
& \leq 3 d \Omega_{1}(h, \tau), \quad i=1,3 \text { if } P_{0} \in D^{0 h} \gamma_{\tau}
\end{aligned}
$$

where $\Omega_{1}$ is as given in (52) and $d$ is the constant given in (55). When $P_{0} \in D^{* h} \gamma_{\tau}$ results

$$
\begin{aligned}
\left|p_{i h}^{2^{n d}}\left(u_{h, \tau}\right)-p_{i h}^{2^{n d}}(u)\right| & \leq \frac{1}{3 h}\left(9 \frac{h}{2} d \Omega_{1}(h, \tau)+\frac{3 h}{2} d \Omega_{1}(h, \tau)\right) \\
& \leq 2 d \Omega_{1}(h, \tau), \quad i=1,3 \text { if } P_{0} \in D^{* h} \gamma_{\tau} .
\end{aligned}
$$

Thus, we obtain (77).

Lemma 4. The following inequality

$$
\max _{S_{T}^{h} \gamma_{1} \cup S_{T}^{h} \gamma_{3}}\left|p_{i h}^{2^{n d}}\left(u_{h, \tau}\right)-p_{i}\right| \leq M_{1} h^{2}+3 d \Omega_{1}(h, \tau), \quad i=1,3,
$$

holds true for $r=\frac{\omega \tau}{h^{2}} \leq \frac{3}{7}$ where $u_{h, \tau}$ is the solution of the difference problem in Stage $\mathbf{1}\left(H^{2 n d}\right)$ and $M_{1}=\frac{1}{3} \max _{\bar{Q}_{T}}\left|\frac{\partial^{3} u}{\partial x_{1}^{3}}\right|$ and $\Omega_{1}$ and $d$ are as given in (52) and (55), respectively.

Proof. From the assumption that the exact solution $u \in C_{x, t}^{7+\alpha, \frac{7+\alpha}{2}}\left(\bar{Q}_{T}\right)$, at the end points $\left(0, \eta \frac{\sqrt{3}}{2} h, k \tau\right) \in S_{T}^{h} \gamma_{1}$ and $\left(a_{1}, \eta \frac{\sqrt{3}}{2} h, k \tau\right) \in S_{T}^{h} \gamma_{3}$ of each line segment

$$
\left[\left(x_{1}, \eta \frac{\sqrt{3}}{2} h, k \tau\right): 0 \leq x_{1} \leq a_{1}, 0 \leq x_{2}=\eta \frac{\sqrt{3}}{2} h \leq a_{2}, 0 \leq t=k \tau \leq T\right]
$$

difference formulas (73) and (74) give the second order approximation of $\frac{\partial u}{\partial x_{1}}$, respectively. From the truncation error formula (see [39]) it follows that

$$
\max _{S_{T}^{h} \gamma_{1} \cup S_{T}^{h} \gamma_{3}}\left|p_{i h}^{2^{n d}}(u)-p_{i}\right| \leq \frac{h^{2}}{3} \max _{\bar{Q}_{T}}\left|\frac{\partial^{3} u}{\partial x_{1}^{3}}\right|, i=1,3 \text { if } P_{0} \in D^{0 h} \gamma_{\tau} .
$$

Analogously,

$$
\max _{S_{T}^{h} \gamma_{1} \cup S_{T}^{h} \gamma_{3}}\left|p_{i h}^{2^{n d}}(u)-p_{i}\right| \leq \frac{h^{2}}{8} \max _{\bar{Q}_{T}}\left|\frac{\partial^{3} u}{\partial x_{1}^{3}}\right|, i=1,3 \text { if } P_{0} \in D^{* h} \gamma_{\tau} .
$$

Using Lemma 3 and the estimations (81) and (82) follows (80).

We construct the following difference problem for the numerical solution of BVP(2) and denote this stage as 


$$
\begin{aligned}
& \text { Stage } 2\left(H^{2 n d}\left(\frac{\partial u}{\partial x_{1}}\right)\right) \\
& \Theta_{h, \tau} v_{h, \tau}^{k+1}=\Lambda_{h, \tau} v_{h, \tau}^{k}+D_{x_{1}} \psi \text { on } D^{0 h} \gamma_{\tau} \text {, } \\
& \Theta_{h, \tau}^{*} \tau_{h, \tau}^{k+1}=\Lambda_{h, \tau}^{*} \tau_{h, \tau}^{k}+\Gamma_{h, \tau}^{*} p_{1 h}^{2^{n d}}+D_{x_{1}} \psi^{*} \text { on } D^{* l h} \gamma_{\tau} \text {, } \\
& \Theta_{h, \tau}^{*} \tau_{h, \tau}^{k+1}=\Lambda_{h, \tau}^{*} v_{h, \tau}^{k}+\Gamma_{h, \tau}^{*} p_{3 h}^{2^{n d}}+D_{x_{1}} \psi^{*} \text { on } D^{* r h} \gamma_{\tau} \text {, } \\
& v_{h, \tau}=p_{i h}^{2^{n d}}\left(u_{h, \tau}\right) \text { on } S_{T}^{h} \gamma_{i}, i=1,3, \\
& v_{h, \tau}=p_{i h} \text { on } S_{T}^{h} \gamma_{i}, i=2,4,5 \text {, }
\end{aligned}
$$

where $p_{1 h}^{2^{n d}}, p_{3 h}^{2^{n d}}$, and $p_{i h}, i=2,4,5$ are defined by (73)-(76) and the operators $\Theta_{h, \tau}$, $\Lambda_{h, \tau}, \Theta_{h, \tau}^{*}, \Lambda_{h, \tau}^{*}$ and $\Gamma_{h, \tau}^{*}$ are the operators given in (16)-(20), respectively. Additionally,

$$
\begin{aligned}
D_{x_{1}} \psi & =\partial_{x_{1}} f_{P_{0}}^{k+\frac{1}{2}} \\
D_{x_{1}} \psi^{*} & =\partial_{x_{1}} f_{P_{0}}^{k+\frac{1}{2}}-\frac{1}{6} \partial_{x_{1}} f_{P_{A}}^{k+\frac{1}{2}},
\end{aligned}
$$

Let

$$
\varepsilon_{h, \tau}^{v}=v_{h, \tau}-v \text { on } \overline{D^{h} \gamma_{\tau}}
$$

where $v=\frac{\partial u}{\partial x_{1}}$. From (83)-(87) and (90), we have

$$
\begin{aligned}
\Theta_{h, \tau} \varepsilon_{h, \tau}^{v, k+1} & =\Lambda_{h, \tau} \varepsilon_{h, \tau}^{v, k}+\Psi_{1}^{v, k} \text { on } D^{0 h} \gamma_{\tau} \\
\Theta_{h, \tau^{*}}^{*} \varepsilon_{h, \tau}^{v, k+1} & =\Lambda_{h, \tau}^{*} \varepsilon_{h, \tau}^{v, k}+\Gamma_{h, \tau}^{*} \varepsilon_{h, \tau}^{* v}+\Psi_{2}^{v, k} \text { on } D^{* h} \gamma_{\tau}, \\
\varepsilon_{h, \tau}^{v} & =0 \text { on } S_{T}^{h} \gamma_{i}, i=2,4,5, \\
\varepsilon_{h, \tau}^{v} & =\varepsilon_{h, \tau}^{* v}=p_{i h}^{2^{n d}}\left(u_{h, \tau}\right)-p_{i} \text { on } S_{T}^{h} \gamma_{i}, i=1,3,
\end{aligned}
$$

where

$$
\begin{aligned}
& \Psi_{1}^{v, k}=\Lambda_{h, \tau} v^{k}-\Theta_{h, \tau} v^{k+1}+D_{x_{1}} \psi, \\
& \Psi_{2}^{v, k}=\Lambda_{h, \tau}^{*} v^{k}-\Theta_{h, \tau}^{*} v^{k+1}+\Gamma_{h, \tau}^{*} p_{i}+D_{x_{1}} \psi^{*}, i=1,3 .
\end{aligned}
$$

Let

$$
\begin{aligned}
& \theta_{1}=\max \left\{\max _{\bar{Q}_{T}}\left|\frac{\partial^{4} v}{\partial x_{1}^{4}}\right|, \overline{m a x}_{T}\left|\frac{\partial^{4} v}{\partial x_{2}^{4}}\right|, \overline{\bar{Q}}_{T}\left|\frac{\partial^{4} v}{\partial x_{1}^{2} \partial x_{2}^{2}}\right|\right\}, \\
& \sigma_{1}=\max \left\{\max _{\bar{Q}_{T}}\left|\frac{\partial^{3} v}{\partial t^{3}}\right|, \max _{\bar{Q}_{T}}\left|\frac{\partial^{4} v}{\partial x_{2}^{2} \partial t^{2}}\right|, \max _{\bar{Q}_{T}}\left|\frac{\partial^{4} v}{\partial x_{1}^{2} \partial t^{2}}\right|\right\},
\end{aligned}
$$

and

$$
\begin{aligned}
\theta & =\max \left\{\theta_{1}, \frac{40 M_{1}}{3}+12 d \omega \alpha^{*}\right\}, \\
\sigma & =\max \left\{\sigma_{1}, 3 d \beta^{*}\right\},
\end{aligned}
$$

where $\alpha^{*}, \beta^{*}$ are as given in (53), (54), respectively, and $M_{1}$ is as given in (80).

Theorem 2. The implicit scheme given in Stage $2\left(\frac{\partial u}{\partial x_{1}}\right)$ is unconditionally stable.

Proof. The obtained algebraic linear system of Equations (83)-(87) can be given in matrix form:

$$
A \widetilde{v}^{k+1}=B \widetilde{v}^{k}+\tau q_{v}^{k}
$$


$k=0,1, \ldots, M^{\prime}-1$, where $A$ and $B$ are the matrices given in (22) and $\widetilde{v}^{k}, q_{v}^{k} \in R^{N}$. On the basis of the assumption that the exact solution $v$ of the $\mathbf{B V P}(2)$ belongs to $C_{x, t}^{6+\alpha, 3+\frac{\alpha}{2}}\left(\bar{Q}_{T}\right)$ and using Lemma 1 and by induction we get

$$
\begin{aligned}
\left\|\widetilde{v}^{k+1}\right\|_{2} & \leq\left\|A^{-1} B\right\|_{2}\left\|\widetilde{v}^{k}\right\|_{2}+\tau\left\|A^{-1}\right\|_{2}\left\|q_{v}^{k}\right\|_{2} \\
& \leq\left\|\widetilde{v}^{0}\right\|_{2}+\tau \sum_{k^{\prime}=0}^{k}\left\|q_{v}^{k^{\prime}}\right\|_{2} .
\end{aligned}
$$

Thus, Lax and Richtmyer sufficient condition for stability given in Theorem 1 of [33] is satisfied and the scheme is unconditionally stable.

Theorem 3. The solution $v_{h, \tau}$ of the finite difference problem given in Stage $2\left(H^{2 n d}\left(\frac{\partial u}{\partial x_{1}}\right)\right)$ satisfies

$$
\frac{\max }{D^{h} \gamma_{\tau}}\left|v_{h, \tau}-v\right| \leq \frac{\sigma}{12}(1+6 \omega)(T+1) \tau^{2}+\frac{3 \theta}{40} h^{2}\left(1+a_{1}^{2}+a_{2}^{2}\right),
$$

for $r=\frac{\omega \tau}{h^{2}} \leq \frac{3}{7}$ where $\theta, \sigma$ are as given in (97), (98), respectively, and $v=\frac{\partial u}{\partial x_{1}}$ is the exact solution of $B V P(2)$.

Proof. Consider the auxiliary system

$$
\begin{aligned}
\Theta_{h, \tau} \widehat{\varepsilon}_{h, \tau}^{v, k+1} & =\Lambda_{h, \tau} \widehat{\varepsilon}_{h, \tau}^{v, k}+\Omega_{2}\left(x_{1}\right) \text { on } D^{0 h} \gamma_{\tau}, \\
\Theta_{h, \tau}^{*} \widehat{\varepsilon}_{h, \tau}^{v, k+1} & =\Lambda_{h, \tau}^{*} \widehat{\varepsilon}_{h, \tau}^{v, k}+\Gamma_{h, \tau}^{*} \widehat{\varepsilon}_{h, \tau}^{v *}+\Omega_{2}\left(x_{1}\right)-\frac{1}{6} \Omega_{2}(\widehat{p}) \text { on } D^{* h} \gamma_{\tau}, \\
\widehat{\varepsilon}_{h, \tau}^{v} & =0 \text { on } S_{T}^{h} \gamma_{i}, i=2,4,5, \\
\widehat{\varepsilon}_{h, \tau}^{v} & =\widehat{\varepsilon}_{h, \tau}^{v *}=p_{i h}^{2^{n d}}\left(u_{h, \tau}\right)-p_{i} \text { on } S_{T}^{h} \gamma_{i}, i=1,3,
\end{aligned}
$$

where

$$
\begin{aligned}
\Omega_{2}\left(x_{1}\right) & =\frac{\sigma}{24 a_{1}}(1+6 \omega) \tau^{2}\left(2 a_{1}-x_{1}\right)+\frac{3 \theta \omega}{10} h^{2}, \\
& \geq \frac{\sigma}{24}(1+6 \omega) \tau^{2}+\frac{3 \theta \omega}{10} h^{2} \geq\left|\Psi_{1}^{v, k}\right|, \\
\Omega_{2}\left(x_{1}\right)-\frac{1}{6} \Omega_{2}(\widehat{p}) & =\left\{\begin{array}{l}
(1+6 \omega) \tau^{2}\left(\frac{5}{72}-\frac{h}{48 a_{1}}\right)+\frac{\theta \omega}{4} h^{2} \text { if } P_{0} \in D^{* l h} \gamma_{\tau} \\
(1+6 \omega) \tau^{2}\left(\frac{5}{144}+\frac{h}{48 a_{1}}\right)+\frac{\theta \omega}{4} h^{2} \text { if } P_{0} \in D^{* r h} \gamma_{\tau} \\
\geq\left|\Psi_{2}^{v, k}\right|,
\end{array}\right.
\end{aligned}
$$

and $x_{1}=\frac{h}{2}$ and $\hat{p}=0$ if $P_{0} \in D^{* h h} \gamma_{\tau}$ and $x_{1}=a_{1}-\frac{h}{2}, \widehat{p}=a_{1}$ if $P_{0} \in D^{* r h} \gamma_{\tau}$. We take the majorant function

$$
\bar{\varepsilon}^{v}\left(x_{1}, x_{2}, t\right)=\bar{\varepsilon}_{1}^{v}\left(x_{1}, x_{2}, t\right)+\bar{\varepsilon}_{2}^{v}\left(x_{1}, x_{2}, t\right),
$$

where

$$
\begin{aligned}
& \bar{\varepsilon}_{1}^{v}\left(x_{1}, x_{2}, t\right)=\frac{\sigma \tau^{2}}{24 a_{1}}(1+6 \omega)(t+1)\left(2 a_{1}-x_{1}\right) \geq 0 \text { on } \overline{D^{h} \gamma_{\tau}} \\
& \bar{\varepsilon}_{2}^{v}\left(x_{1}, x_{2}, t\right)=\frac{3 \theta}{40} h^{2}\left(1+a_{1}^{2}+a_{2}^{2}-x_{1}^{2}-x_{2}^{2}\right) \geq 0 \text { on } \overline{D^{h} \gamma_{\tau}}
\end{aligned}
$$


The function in (108) satisfies the difference problem

$$
\begin{aligned}
\Theta_{h, \tau} \bar{\varepsilon}_{h, \tau}^{v, k+1} & =\Lambda_{h, \tau} \bar{\varepsilon}_{h, \tau}^{v, k}+\Omega_{2}\left(x_{1}\right) \text { on } D^{0 h} \gamma_{\tau}, \\
\Theta_{h, \tau}^{*} \bar{\varepsilon}_{h, \tau}^{,, k+1} & =\Lambda_{h, \tau}^{*} \bar{\varepsilon}_{h, \tau}^{v, k}+\Gamma_{h, \tau}^{*} \bar{\varepsilon}_{h, \tau}^{v *}+\Omega_{2}\left(x_{1}\right)-\frac{1}{6} \Omega_{2}(\widehat{p}) \text { on } D^{* h} \gamma_{\tau}, \\
\bar{\varepsilon}_{h, \tau}^{v} & =\bar{\varepsilon}_{h, \tau}^{v *}=\bar{\varepsilon}_{1}^{v}\left(0, x_{2}, t\right)+\bar{\varepsilon}_{2}^{v}\left(0, x_{2}, t\right) \text { on } S_{T}^{h} \gamma_{1}, \\
\bar{\varepsilon}_{h, \tau}^{v} & =\bar{\varepsilon}_{1}^{v}\left(x_{1}, 0, t\right)+\bar{\varepsilon}_{2}^{v}\left(x_{1}, 0, t\right) \text { on } S_{T}^{h} \gamma_{2}, \\
\bar{\varepsilon}_{h, \tau}^{v} & =\bar{\varepsilon}_{h, \tau}^{v *}=\bar{\varepsilon}_{1}^{v}\left(a_{1}, x_{2}, t\right)+\bar{\varepsilon}_{2}^{v}\left(a_{1}, x_{2}, t\right) \text { on } S_{T}^{h} \gamma_{3}, \\
\bar{\varepsilon}_{h, \tau}^{v} & =\bar{\varepsilon}_{1}^{v}\left(x_{1}, a_{2}, t\right)+\bar{\varepsilon}_{2}^{v}\left(x_{1}, a_{2}, t\right) \text { on } S_{T}^{h} \gamma_{4}, \\
\bar{\varepsilon}_{h, \tau}^{v} & =\bar{\varepsilon}_{1}^{v}\left(x_{1}, x_{2}, 0\right)+\bar{\varepsilon}_{2}^{v}\left(x_{1}, x_{2}, 0\right) \text { on } S_{T}^{h} \gamma_{5} .
\end{aligned}
$$
form as

The algebraic system of Equations (102)-(105) and (111)-(117) can be written in matrix

$$
\begin{aligned}
& A \widehat{\varepsilon}^{v, k+1}=B \widehat{\varepsilon}^{v, k}+\tau \widehat{e}^{v, k}, \\
& A \bar{\varepsilon}^{v, k+1}=B \bar{\varepsilon}^{v, k}+\tau \bar{e}^{v, k},
\end{aligned}
$$

respectively, for $k=0, \ldots, M^{\prime}-1$, where $A, B$ are matrices as given in (22) and $\widehat{\varepsilon}^{v, k}, \bar{\varepsilon}^{v, k}$, $\widehat{e}^{v, k}, \bar{e}^{v, k} \in R^{N}$. Using (106)-(117), we have $\bar{\varepsilon}^{v, 0} \geq 0$, and $\bar{e}^{v, k} \geq 0$, and $\left|\widehat{e}^{v, k}\right| \leq \bar{e}^{v, k}$ for $k=0, \ldots, M^{\prime}-1$, and $\left|\widehat{\varepsilon}^{v, 0}\right| \leq \bar{\varepsilon}^{v, 0}$. Then, on the basis of Lemma 2, we get $\left|\widehat{\varepsilon}^{v, k+1}\right| \leq \bar{\varepsilon}^{v, k+1}$ for $k=0, \ldots, M^{\prime}-1$. From

$$
\begin{aligned}
\bar{\varepsilon}^{v}\left(x_{1}, x_{2}, t\right) & \leq \bar{\varepsilon}^{v}(0,0, T) \\
& =\frac{\sigma}{12}(1+6 \omega)(T+1) \tau^{2}+\frac{3 \theta}{40} h^{2}\left(1+a_{1}^{2}+a_{2}^{2}\right)
\end{aligned}
$$

and using (106) and (107) follows (101).

\section{Second Order Approximation of $\frac{\partial u}{\partial x_{2}}$ on Hexagonal grids}

Let the BVP(1) be given, then we use the notations $\partial_{x_{2}} f_{P_{0}}^{k+\frac{1}{2}}=\left.\frac{\partial f}{\partial x_{2}}\right|_{\left(x_{1}, x_{2}, t+\frac{\tau}{2}\right)}$ and $\partial_{x_{2}} f_{P_{A}}^{k+\frac{1}{2}}=\left.\frac{\partial f}{\partial x_{2}}\right|_{\left(\widehat{p}, x_{2}, t+\frac{\tau}{2}\right)}$ and denote $q_{i}=\frac{\partial u}{\partial x_{2}}$ on $S_{T} \gamma_{i}, i=1,2, \ldots, 5$ and setup the next boundary value problem for $z=\frac{\partial u}{\partial x_{2}}$,

BVP(3):

$$
\begin{aligned}
L z & =\frac{\partial f\left(x_{1}, x_{2}, t\right)}{\partial x_{2}} \text { on } Q_{T}, \\
z\left(x_{1}, x_{2}, t\right) & =q_{i} \text { on } S_{T} \gamma_{i}, i=1,2, \ldots, 5,
\end{aligned}
$$

where $L$ is the operator in (72) and $f\left(x_{1}, x_{2}, t\right)$ is the given function in (2). We assume that the solution $z \in C_{x, t}^{6+\alpha, 3+\frac{\alpha}{2}}\left(\bar{Q}_{T}\right)$ and take 


$$
\begin{aligned}
q_{2 h}^{2^{\text {nd }}}= & \frac{1}{2 \sqrt{3} h}\left(-3 u\left(x_{1}, 0, t\right)+4 u_{h, \tau}\left(x_{1}, \sqrt{3} h, t\right)\right. \\
& \left.-u_{h, \tau}\left(x_{1}, 2 \sqrt{3} h, t\right)\right) \text { on } S_{T}^{h} \gamma_{2}, \\
q_{4 h}^{2^{n d}}= & \frac{1}{2 \sqrt{3} h}\left(3 u\left(x_{1}, a_{2}, t\right)-4 u_{h, \tau}\left(x_{1}, a_{2}-\sqrt{3} h, t\right)\right. \\
& \left.+u_{h, \tau}\left(x_{1}, a_{2}-2 \sqrt{3} h, t\right)\right) \text { on } S_{T}^{h} \gamma_{4}, \\
q_{i h}= & \frac{\partial \phi\left(x_{1}, x_{2}, t\right)}{\partial x_{2}} \text { on } S_{T}^{h} \gamma_{i}, i=1,3, \\
q_{5 h}= & \frac{\partial \varphi\left(x_{1}, x_{2}\right)}{\partial x_{2}} \text { on } S_{T}^{h} \gamma_{5},
\end{aligned}
$$

and $\varphi\left(x_{1}, x_{2}\right)$ given in (3), $\phi\left(x_{1}, x_{2}, t\right)$ given in (4) are the initial and boundary functions, respectively, $u_{h, \tau}$ is the solution of the difference problem in Stage $\mathbf{1}\left(H^{2 n d}\right)$.

Lemma 5. The following inequality holds

$$
\left|q_{i h}^{2^{n d}}\left(u_{h, \tau}\right)-q_{i h}^{2^{n d}}(u)\right| \leq 3 d \Omega_{1}(h, \tau), \quad i=2,4,
$$

for $r=\frac{\omega \tau}{h^{2}} \leq \frac{3}{7}$, where $u$ is the solution of the boundary value Problems (2)-(4) and $u_{h, \tau}$ is the solution of the difference Problems (10)-(13) in Stage $\mathbf{1}\left(H^{2 n d}\right)$ and $\Omega_{1}(h, \tau)$ and $d$ are as given in (52) and (55), respectively.

Proof. Taking into consideration Theorem 1, and using (51), (122), and (123), we have

$$
\begin{aligned}
\left|q_{i h}^{2 n d}\left(u_{h, \tau}\right)-q_{i h}^{2^{n d}}(u)\right| & \leq \frac{1}{2 \sqrt{3} h}\left(4 \sqrt{3} h d \Omega_{1}(h, \tau)+2 \sqrt{3} h d \Omega_{1}(h, \tau)\right) \\
& \leq 3 d \Omega_{1}(h, \tau), \quad i=2,4
\end{aligned}
$$

thus, we obtain (126).

Lemma 6. The following inequality is true

$$
\max _{S_{T}^{h} \gamma_{2} \cup S_{T}^{h} \gamma_{4}}\left|q_{i h}^{2^{n d}}\left(u_{h, \tau}\right)-q_{i}\right| \leq M_{2} h^{2}+3 d \Omega_{1}(h, \tau), i=2,4,
$$

for $r=\frac{\omega \tau}{h^{2}} \leq \frac{3}{7}$, where $M_{2}=\max _{\bar{Q}_{T}}\left|\frac{\partial^{3} u}{\partial x_{2}^{3}}\right|$ and $u_{h, \tau}$ is the solution of the difference problem in Stage $\mathbf{1}\left(H^{2 n d}\right)$ and $\Omega_{1}(h, \tau)$ and $d$ are as given in (52) and (55), respectively.

Proof. From the assumption that the exact solution $u \in C_{x, t}^{7+\alpha, \frac{7+\alpha}{2}}\left(\bar{Q}_{T}\right)$, at the end points $(\vartheta h, 0, k \tau) \in S_{T}^{h} \gamma_{2}$ and $\left(\vartheta h, a_{2}, k \tau\right) \in S_{T}^{h} \gamma_{4}$ of each line segment

$$
\left[\left(\vartheta h, x_{2}, k \tau\right): 0 \leq x_{1}=\vartheta h \leq a_{1}, 0 \leq x_{2} \leq a_{2}, 0 \leq t=k \tau \leq T\right],
$$

difference formulas (122) and (123) give the second order approximation of $\frac{\partial u}{\partial x_{2}}$, respectively. From the truncation error formula (see [39]), it follows that

$$
\max _{S_{T}^{h} \gamma_{2} \cup S_{T}^{h} \gamma_{4}}\left|q_{i h}^{2 n d}(u)-q_{i}\right| \leq h^{2} \max _{\bar{Q}_{T}}\left|\frac{\partial^{3} u}{\partial x_{2}^{3}}\right|, i=2,4 .
$$


Taking $M_{2}=\max _{\bar{Q}_{T}}\left|\frac{\partial^{3} u}{\partial x_{2}^{3}}\right|$ and using Lemma 5 and the estimation (127) and (129) follows (128).

We construct the following difference problem for the numerical solution of BVP(3) and denote this stage as

$$
\begin{aligned}
& \text { Stage } 2\left(H^{2 n d}\left(\frac{\partial u}{\partial x_{2}}\right)\right) \\
& \qquad \begin{aligned}
\Theta_{h, \tau} z_{h, \tau}^{k+1} & =\Lambda_{h, \tau} z_{h, \tau}^{k}+D_{x_{2}} \psi \text { on } D^{0 h} \gamma_{\tau}, \\
\Theta_{h, \tau}^{*} z_{h, \tau}^{k+1} & =\Lambda_{h, \tau}^{*} z_{h, \tau}^{k}+\Gamma_{h, \tau}^{*} q_{1 h}+D_{x_{2}} \psi^{*} \text { on } D^{* l h} \gamma_{\tau}, \\
\Theta_{h, \tau}^{*} z_{h, \tau}^{k+1} & =\Lambda_{h, \tau}^{*} z_{h, \tau}^{k}+\Gamma_{h, \tau}^{*} q_{3 h}+D_{x_{2}} \psi^{*} \text { on } D^{* r h} \gamma_{\tau}, \\
z_{h, \tau} & =q_{i h}^{2 n d}\left(u_{h, \tau}\right) \text { on } S_{T}^{h} \gamma_{i}, i=2,4, \\
z_{h, \tau} & =q_{i h} \text { on } S_{T}^{h} \gamma_{i}, i=1,3,5,
\end{aligned}
\end{aligned}
$$

where $q_{2 h}^{2 n d}, q_{4 h}^{2 n d}$, and $q_{i h}, i=1,3,5$ are defined by (122)-(125) and the operators $\Theta_{h, \tau}$, $\Lambda_{h, \tau}, \Theta_{h, \tau}^{*}, \Lambda_{h, \tau}^{*}$, and $\Gamma_{h, \tau}^{*}$ are the operators given in (16)-(20), respectively. In addition,

$$
\begin{aligned}
D_{x_{2}} \psi & =\partial_{x_{2}} f_{P_{0}}^{k+\frac{1}{2}} \\
D_{x_{2}} \psi^{*} & =\partial_{x_{2}} f_{P_{0}}^{k+\frac{1}{2}}-\frac{1}{6} \partial_{x_{2}} f_{P_{A}}^{k+\frac{1}{2}} .
\end{aligned}
$$

Let

$$
\varepsilon_{h, \tau}^{z}=z_{h, \tau}-z \text { on } \overline{D^{h} \gamma_{\tau}} .
$$

From (130)-(134) and (137), we have

$$
\begin{aligned}
\Theta_{h, \tau} \varepsilon_{h, \tau}^{z, k+1} & =\Lambda_{h, \tau} \varepsilon_{h, \tau}^{z, k}+\Psi_{1}^{z, k} \text { on } D^{0 h} \gamma_{\tau}, \\
\Theta_{h, \tau}^{*} \varepsilon_{h, \tau}^{z, k+1} & =\Lambda_{h, \tau}^{*} \varepsilon_{h, \tau}^{z, k}+\Psi_{2}^{z, k} \text { on } D^{* h} \gamma_{\tau}, \\
\varepsilon_{h, \tau}^{z} & =0 \text { on } S_{T}^{h} \gamma_{i}, i=1,3,5, \\
\varepsilon_{h, \tau}^{z} & =q_{i h}^{2^{n d}}\left(u_{h, \tau}\right)-q_{i} \text { on } S_{T}^{h} \gamma_{i,}, i=2,4,
\end{aligned}
$$

where $q_{i h}$ are defined by (122)-(125) and

$$
\begin{aligned}
& \Psi_{1}^{z, k}=\Lambda_{h, \tau} z^{k}-\Theta_{h, \tau} z^{k+1}+D_{x_{2}} \psi, \\
& \Psi_{2}^{z, k}=\Lambda_{h, \tau}^{*} z^{k}-\Theta_{h, \tau}^{*} z^{k+1}+\Gamma_{h, \tau}^{*} q_{i}+D_{x_{2}} \psi^{*}, i=1,3 .
\end{aligned}
$$

Let

$$
\begin{aligned}
& \kappa_{1}=\max \left\{\max _{\overline{\bar{Q}}_{T}}\left|\frac{\partial^{4} z}{\partial x_{1}^{4}}\right|, \overline{\max }_{T}\left|\frac{\partial^{4} z}{\partial x_{2}^{4}}\right|, \overline{\max }_{T}\left|\frac{\partial^{4} z}{\partial x_{1}^{2} \partial x_{2}^{2}}\right|\right\}, \\
& \delta_{1}=\max \left\{\max _{\bar{Q}_{T}}\left|\frac{\partial^{3} z}{\partial t^{3}}\right|, \max _{\bar{Q}_{T}}\left|\frac{\partial^{4} z}{\partial x_{2}^{2} \partial t^{2}}\right|, \max _{\bar{Q}_{T}}\left|\frac{\partial^{4} z}{\partial x_{1}^{2} \partial t^{2}}\right|\right\},
\end{aligned}
$$

and

$$
\begin{aligned}
\kappa & =\max \left\{\kappa_{1}, \frac{40 M_{2}}{3}+12 d \omega \alpha^{*}\right\}, \\
\delta & =\max \left\{\delta_{1}, 3 d \beta^{*}\right\},
\end{aligned}
$$

$\alpha^{*}, \beta^{*}$ are as given in (53), (54), respectively, and $M_{2}$ is the constant given in Lemma 6 and $z$ is the solution of $\mathbf{B V P}(3)$. 
Theorem 4. The implicit scheme given in Stage $2\left(H^{2 n d}\left(\frac{\partial u}{\partial x_{2}}\right)\right)$ is unconditionally stable.

Proof. The obtained algebraic linear system of Equations (130)-(134) can be given in matrix form:

$$
A \widetilde{z}^{k+1}=B \widetilde{z}^{k}+\tau q_{z}^{k}
$$

for $k=0,1, \ldots, M^{\prime}-1$, where, $A, B$ are as given in (22) and $\widetilde{z}^{k}, q_{z}^{k} \in R^{N}$. On the basis of the assumption that the exact solution $z$ of the BVP(3) belongs to $C_{x, t}^{6+\alpha, 3+\frac{\alpha}{2}}\left(\bar{Q}_{T}\right)$ and using Lemma 1 and induction we get

$$
\begin{aligned}
\left\|\widetilde{z}^{k+1}\right\|_{2} & \leq\left\|A^{-1} B\right\|_{2}\left\|\widetilde{z}^{k}\right\|_{2}+\tau\left\|A^{-1}\right\|_{2}\left\|q_{z}^{k}\right\|_{2} \\
& \leq\left\|\widetilde{z}^{0}\right\|_{2}+\tau \sum_{k^{\prime}=0}^{k}\left\|q_{z}^{k^{\prime}}\right\|_{2} \cdot
\end{aligned}
$$

Therefore, the scheme is unconditionally stable.

Theorem 5. The solution $z_{h, \tau}$ of the finite difference problem given in Stage $2\left(H^{2 n d}\left(\frac{\partial u}{\partial x_{2}}\right)\right)$ satisfies

$$
\frac{\max }{D^{h} \gamma_{\tau}}\left|z_{h, \tau}-z\right| \leq \frac{\delta}{12}(1+6 \omega)(T+1) \tau^{2}+\frac{3 \kappa}{40}\left(1+a_{1}^{2}+a_{2}^{2}\right) h^{2}
$$

for $r=\frac{\omega \tau}{h^{2}} \leq \frac{3}{7}$, where $\kappa, \delta$ are as given in (146), (147) respectively and $z=\frac{\partial u}{\partial x_{2}}$ is the exact solution of $\boldsymbol{B V P ( 3 )}$.

Proof. Consider the auxiliary system

$$
\begin{aligned}
\Theta_{h, \tau} \widehat{\varepsilon}_{h, \tau}^{z, k+1} & =\Lambda_{h, \tau} \widehat{\varepsilon}_{h, \tau}^{z, k}+\Omega_{3}\left(x_{2}\right) \text { on } D^{0 h} \gamma_{\tau}, \\
\Theta_{h, \tau}^{*} \widehat{\varepsilon}_{h, \tau}^{z, k+1} & =\Lambda_{h, \tau}^{*} \widehat{\varepsilon}_{h, \tau}^{z, k}+\frac{5}{6} \Omega_{3}\left(x_{2}\right) \text { on } D^{* h} \gamma_{\tau} \\
\widehat{\varepsilon}_{h, \tau}^{z} & =0 \text { on } S_{T}^{h} \gamma_{i}, i=1,3,5, \\
\widehat{\varepsilon}_{h, \tau}^{z} & =q_{i h}^{2 n}\left(u_{h, \tau}\right)-q_{i} \text { on } S_{T}^{h} \gamma_{i}, i=2,4,
\end{aligned}
$$

where $q_{2 h}^{2^{n d}}, q_{4 h}^{2^{\text {nd }}}, q_{i h}, i=1,3,5$, are defined by (122)-(125) and

$$
\begin{aligned}
\Omega_{3}\left(x_{2}\right) & =\frac{\delta}{24 a_{2}}(1+6 \omega) \tau^{2}\left(2 a_{2}-x_{2}\right)+\frac{3 \kappa \omega}{10} h^{2} \\
& \geq \frac{\delta}{24}(1+6 \omega) \tau^{2}+\frac{3 \kappa \omega}{10} h^{2} \geq\left|\Psi_{1}^{z, k}\right|, \\
\frac{5}{6} \Omega_{3}\left(x_{2}\right) & =\frac{5 \delta}{144 a_{2}}(1+6 \omega) \tau^{2}\left(2 a_{2}-x_{2}\right)+\frac{\kappa \omega}{4} h^{2} \\
& \geq \frac{5 \delta}{144}(1+6 \omega) \tau^{2}+\frac{\kappa \omega}{4} h^{2} \geq\left|\Psi_{2}^{z, k}\right| .
\end{aligned}
$$

We take the majorant function

$$
\bar{\varepsilon}^{z}\left(x_{1}, x_{2}, t\right)=\bar{\varepsilon}_{1}^{z}\left(x_{1}, x_{2}, t\right)+\bar{\varepsilon}_{2}^{z}\left(x_{1}, x_{2}, t\right),
$$

where

$$
\begin{aligned}
& \bar{\varepsilon}_{1}^{z}\left(x_{1}, x_{2}, t\right)=\frac{\delta}{24 a_{2}} \tau^{2}(1+6 \omega)(t+1)\left(2 a_{2}-x_{2}\right) \geq 0 \text { on } \overline{D^{h} \gamma_{\tau}} \\
& \bar{\varepsilon}_{2}^{z}\left(x_{1}, x_{2}, t\right)=\frac{3 \kappa}{40} h^{2}\left(1+a_{1}^{2}+a_{2}^{2}-x_{1}^{2}-x_{2}^{2}\right) \geq 0 \text { on } \overline{D^{h} \gamma_{\tau}}
\end{aligned}
$$


The majorant function in (157) satisfies the difference problem

$$
\begin{aligned}
\Theta_{h, \tau} \bar{\varepsilon}_{h, \tau}^{z, k+1} & =\Lambda_{h, \tau} \bar{\varepsilon}_{h, \tau}^{z, k}+\Omega_{3}\left(x_{2}\right) \text { on } D^{0 h} \gamma_{\tau}, \\
\Theta_{h, \tau}^{*} \bar{\varepsilon}_{h, \tau}^{z, k+1} & =\Lambda_{h, \tau}^{*} \bar{\varepsilon}_{h, \tau}^{z, k}+\Gamma_{h, \tau}^{*} \bar{\varepsilon}_{h, \tau}^{z *}+\frac{5}{6} \Omega_{3}\left(x_{2}\right) \text { on } D^{* h} \gamma_{\tau}, \\
\bar{\varepsilon}_{h, \tau}^{z} & =\bar{\varepsilon}_{h, \tau}^{z *}=\bar{\varepsilon}_{1}^{z}\left(0, x_{2}, t\right)+\bar{\varepsilon}_{2}^{z}\left(0, x_{2}, t\right) \text { on } S_{T}^{h} \gamma_{1}, \\
\bar{\varepsilon}_{h, \tau}^{z} & =\bar{\varepsilon}_{1}^{z}\left(x_{1}, 0, t\right)+\bar{\varepsilon}_{2}^{z}\left(x_{1}, 0, t\right) \text { on } S_{T}^{h} \gamma_{2}, \\
\bar{\varepsilon}_{h, \tau}^{z} & =\bar{\varepsilon}_{h, \tau}^{z_{2}^{*}}=\bar{\varepsilon}_{1}^{z}\left(a_{1}, x_{2}, t\right)+\bar{\varepsilon}_{2}^{z}\left(a_{1}, x_{2}, t\right) \text { on } S_{T}^{h} \gamma_{3}, \\
\bar{\varepsilon}_{h, \tau}^{z} & =\bar{\varepsilon}_{1}^{z}\left(x_{1}, a_{2}, t\right)+\bar{\varepsilon}_{2}^{z}\left(x_{1}, a_{2}, t\right) \text { on } S_{T}^{h} \gamma_{4}, \\
\bar{\varepsilon}_{h, \tau}^{z} & =\bar{\varepsilon}_{1}^{z}\left(x_{1}, x_{2}, 0\right)+\bar{\varepsilon}_{2}^{z}\left(x_{1}, x_{2}, 0\right) \text { on } S_{T}^{h} \gamma_{5} .
\end{aligned}
$$

We write the algebraic system of Equations (151)-(154) and (160)-(166) for fixed $k \geq 0$ in matrix form

$$
\begin{aligned}
& A \widehat{\varepsilon}^{z, k+1}=B \widehat{\varepsilon}^{z, k}+\tau \widehat{e}^{z, k}, \\
& A \bar{\varepsilon}^{z, k+1}=B \bar{\varepsilon}^{z, k}+\tau \bar{e}^{z, k},
\end{aligned}
$$

respectively, where $A, B$ are as given in (22) and $\widehat{\mathcal{\varepsilon}}^{z, k}, \bar{\varepsilon}^{z, k}, \widehat{e}^{z, k}, \bar{e}^{z, k} \in R^{N}$. Using (155)-(166), we get $\bar{e}^{z, k} \geq 0$ and $\left|\widehat{e}^{z, k}\right| \leq \bar{e}^{z, k}$ for $k=0,1, \ldots, M^{\prime}-1$ and $\bar{\varepsilon}^{z, 0} \geq 0,\left|\widehat{\varepsilon}^{z, 0}\right| \leq \bar{\varepsilon}^{z, 0}$. Then, on the basis of Lemma 2 follows $\left|\widehat{\varepsilon}^{z, k+1}\right| \leq \bar{\varepsilon}^{z, k+1}, k=0,1, \ldots, M^{\prime}-1$. From

$$
\begin{aligned}
\bar{\varepsilon}^{z}\left(x_{1}, x_{2}, t\right) & \leq \bar{\varepsilon}^{z}(0,0, T) \\
& =\frac{\delta}{12}(1+6 \omega)(T+1) \tau^{2}+\frac{3 \kappa}{40}\left(1+a_{1}^{2}+a_{2}^{2}\right) h^{2},
\end{aligned}
$$

and using (155), (156) follows (150).

\section{Numerical Results}

A test problem is constructed of which the exact solution is known to show the efficiency of the proposed two stage implicit method. The rectangle $D$ is taken as $D=$ $\left\{\left(x_{1}, x_{2}\right): 0<x_{1}<1,0<x_{2}<\frac{\sqrt{3}}{2}\right\}$, and $t \in[0,1]$. All the computations are performed using Mathematica in double precision on a personal computer with properties AMD Ryzen 7 1800X Eight Core Processor 3.60GHz. To solve the obtained linear algebraic system of equations, we applied incomplete block-matrix factorization of the block tridiagonal stiffness matrices which are symmetric $M$-matrices for the all considered pairs of $(h, \tau)$ using two-step iterative method for matrix inversion. Then these incomplete block-matrix factorizations are used as preconditioners for the conjugate gradient method as given in [34] (see also $[35,36]$ ). We use the following notations in tables and figures:

$H^{2 n d}\left(\frac{\partial u}{\partial x_{1}}\right)$ denotes the proposed two stage implicit method on hexagonal grids for the approximation of the derivative $\frac{\partial u}{\partial x_{1}}$.

$H^{2 n d}\left(\frac{\partial u}{\partial x_{2}}\right)$ denotes the proposed two stage implicit method on hexagonal grids for the approximation of the derivative $\frac{\partial u}{\partial x_{2}}$.

$C T^{H^{2 n d}\left(\frac{\partial u}{\partial x_{1}}\right)}$ presents the Central Processing Unit time in seconds (CPUs) per time level for the method $H^{2 n d}\left(\frac{\partial u}{\partial x_{1}}\right)$.

$C T^{H^{2 n d}\left(\frac{\partial u}{\partial x_{2}}\right)}$ presents the Central Processing Unit time in seconds (CPUs) per time level for the method $H^{2 n d}\left(\frac{\partial u}{\partial x_{2}}\right)$. 
$T C T^{H^{2 n d}\left(\frac{\partial u}{\partial x_{1}}\right)}$ shows the total Central Processing Unit time in seconds required for the solution at $t=1$, by the method $H^{2 n d}\left(\frac{\partial u}{\partial x_{1}}\right)$.

$T C T^{H^{2 n d}\left(\frac{\partial u}{\partial x_{2}}\right)}$ shows the total Central Processing Unit time in seconds required for the solution at $t=1$, by the method $H^{2 n d}\left(\frac{\partial u}{\partial x_{2}}\right)$.

The proposed two stage implicit method for the approximation of the derivatives $\frac{\partial u}{\partial x_{1}}, \frac{\partial u}{\partial x_{2}}$ are denoted as the methods $H^{2 n d}\left(\frac{\partial u}{\partial x_{1}}\right)$, and $H^{2 n d}\left(\frac{\partial u}{\partial x_{2}}\right)$. Additionally, the corresponding solutions are denoted by $v_{2^{-\mu}, 2^{-\lambda}}$, and $z_{2^{-\mu}, 2^{-\lambda}}$, respectively, for $h=2^{-\mu}$ and $\tau=2^{-\lambda}$ where $\mu, \lambda$ are positive integers. On the grid points $\overline{D^{h} \gamma_{\tau}}$, which is the closure of $D^{h} \gamma_{\tau}$ we present the error function $\varepsilon_{h, \tau}$ obtained by $H^{2 n d}\left(\frac{\partial u}{\partial x_{1}}\right)$, and $H^{2 n d}\left(\frac{\partial u}{\partial x_{2}}\right)$ by $\varepsilon^{H^{2 n d}\left(\frac{\partial u}{\partial x_{1}}\right)}$ and by $\varepsilon^{H^{2 n d}\left(\frac{\partial u}{\partial x_{2}}\right)}$, respectively. In addition, maximum norm of the errors $\frac{\max }{D^{h} \gamma_{\tau}}\left|\varepsilon^{H^{2 n d}}\left(\frac{\partial u}{\partial x_{1}}\right)\right|$ and $\frac{\max }{D^{h} \gamma_{\tau}}\left|\varepsilon^{H^{2 n d}\left(\frac{\partial u}{\partial x_{2}}\right)}\right|$ are presented by $\left\|\mathcal{E}^{H^{2 n d}\left(\frac{\partial u}{\partial x_{1}}\right)}\right\|_{\infty}$ and $\left\|\mathcal{E}^{H^{2 n d}\left(\frac{\partial u}{\partial x_{2}}\right)}\right\|_{\infty}$, accordingly. Further, we denote the order of convergence of the approximate solution $v_{2^{-\mu}, 2^{-\lambda}}$ to the exact solution $v=\frac{\partial u}{\partial x_{1}}$ obtained by using the two stage implicit method $H^{2 n d}\left(\frac{\partial u}{\partial x_{1}}\right)$ by

$$
\Re^{H^{2 n d}\left(\frac{\partial u}{\partial x_{1}}\right)}=\frac{\left\|\mathcal{E}^{H^{2 n d}\left(\frac{\partial u}{\partial x_{1}}\right)\left(2^{-\mu}, 2^{-\lambda}\right)}\right\|_{\infty}}{\left\|\varepsilon^{H^{2 n d}\left(\frac{\partial u}{\partial x_{1}}\right)\left(2^{-(\mu+1)}, 2^{-(\lambda+1)}\right)}\right\|_{\infty}} .
$$

Analogously, the order of convergence of the approximate solution $z_{2^{-\mu}, 2^{-\lambda}}$ to the exact solution $z=\frac{\partial u}{\partial x_{2}}$ obtained by using the two stage implicit method $H^{2 n d}\left(\frac{\partial u}{\partial x_{2}}\right)$ is given by

$$
\Re^{H^{2 n d}\left(\frac{\partial u}{\partial x_{2}}\right)}=\frac{\left\|\varepsilon^{H^{2 n d}\left(\frac{\partial u}{\partial x_{2}}\right)\left(2^{-\mu}, 2^{-\lambda}\right)}\right\|_{\infty}}{\left\|\varepsilon^{E^{2 n d}\left(\frac{\partial u}{\partial x_{2}}\right)\left(2^{-(\mu+1)}, 2^{-(\lambda+1)}\right)}\right\|_{\infty} .}
$$

We remark that the values of (170), (171) are $\approx 2^{2}$ showing that the order of convergence of the approximate solution $v_{2^{-\mu}, 2^{-\lambda}}$ to the exact solution $v=\frac{\partial u}{\partial x_{1}}$ and the order of convergence of the approximate solution $z_{2^{-\mu}, 2^{-\lambda}}$ to the exact solution $z=\frac{\partial u}{\partial x_{2}}$ are second order both in the spatial variables $x_{1}, x_{2}$ and in time $t$, accordingly.

Test Problem:

$$
\begin{aligned}
\frac{\partial u}{\partial t} & =0.5\left(\frac{\partial^{2} u}{\partial x_{1}^{2}}+\frac{\partial^{2} u}{\partial x_{2}^{2}}\right)+f\left(x_{1}, x_{2}, t\right) \text { on } Q_{T} \\
u\left(x_{1}, x_{2}, 0\right) & =0.0001\left(x_{1}^{\frac{57}{8}}\left(1-x_{1}\right)+\cos \left(x_{2}^{\frac{57}{8}}\right)\left(\frac{\sqrt{3}}{2}-x_{2}\right)\right) \text { on } \bar{D}, \\
u\left(x_{1}, x_{2}, t\right) & =\widehat{u}\left(x_{1}, x_{2}, t\right) \text { on } S_{T}
\end{aligned}
$$

where 


$$
\begin{aligned}
f\left(x_{1}, x_{2}, t\right)= & 0.00035625\left(t^{\frac{41}{16}}-6.125 x_{1}^{\frac{41}{8}}+8.125 x_{1}^{\frac{49}{8}}\right. \\
& +\left(\sqrt{3} \frac{3249}{912}-7.125 x_{2}\right) x_{2}^{\frac{49}{4}} \cos \left(x_{2}^{\frac{57}{8}}\right) \\
& \left.+\left(\sqrt{3} \frac{2793}{912}-8.125 x_{2}\right) x_{2}^{\frac{41}{8}} \sin \left(x_{2}^{\frac{57}{8}}\right)\right) \\
\widehat{u}\left(x_{1}, x_{2}, t\right)= & 0.0001\left(t^{\frac{57}{16}}+x_{1}^{\frac{57}{8}}\left(1-x_{1}\right)+\cos \left(x_{2}^{\frac{57}{8}}\right)\left(\frac{\sqrt{3}}{2}-x_{2}\right)\right),
\end{aligned}
$$

are the heat source and exact solution. Table 1 demonstrates $C T^{H^{2 n d}\left(\frac{\partial u}{\partial x_{1}}\right)}, T C T^{H^{2 n d}}\left(\frac{\partial u}{\partial x_{1}}\right)$, maximum norm of the errors for $h=2^{-\mu}, \mu=4,5,6,7$ when $\tau=2^{-\lambda}, \lambda=13,14,15,16$, that is $r=\frac{0.5 \tau}{h^{2}} \leq \frac{3}{7}$ and the order of convergence of $v_{h, \tau}$ to the exact derivatives $v=\frac{\partial u}{\partial x_{1}}$ with respect to $h$ and $\tau$ obtained by using the constructed two stage implicit method $H^{2 n d}\left(\frac{\partial u}{\partial x_{1}}\right)$. Table 2 shows $C T^{H^{2 n d}\left(\frac{\partial u}{\partial x_{2}}\right)}, T C T^{H^{2 n d}\left(\frac{\partial u}{\partial x_{2}}\right)}$, maximum norm of the errors for the same pairs of $(h, \tau)$ as in Table 1 and the order of convergence of $z_{h, \tau}$ to the exact derivative $z=\frac{\partial u}{\partial x_{2}}$ with respect to $h$ and $\tau$ obtained by using the constructed two stage implicit method $H^{2 n d}\left(\frac{\partial u}{\partial x_{2}}\right)$. Tables 1 and 2 justify the theoretical results given such that the approximate solutions $v_{h, \tau}$ and $z_{h, \tau}$ of the proposed method converge to the corresponding exact derivatives $v=\frac{\partial u}{\partial x_{1}}$ and $z=\frac{\partial u}{\partial x_{2}}$ with second order both in the spatial variables $x_{1}, x_{2}$ and the time variable $t$ for $r \leq \frac{3}{7}$.

Table 1. Computational time, maximum norm of the errors, and the order of convergence of $v_{h, \tau}$ to the exact derivative $v=\frac{\partial u}{\partial x_{1}}$ when $r=\frac{0.5 \tau}{h^{2}} \leq \frac{3}{7}$.

\begin{tabular}{cccccc}
\hline$r=\frac{0.5 \tau}{h^{2}}$ & $(h, \tau)$ & $C T^{H^{2 n d}\left(\frac{\partial u}{\partial x_{1}}\right)}$ & $T C T^{H^{2 n d}}\left(\frac{\partial u}{\partial x_{1}}\right)$ & $\left\|\varepsilon^{H^{2 n d}}\left(\frac{\partial u}{\partial x_{1}}\right)\right\|_{\infty}$ & $\Re^{H^{2 n d}\left(\frac{\partial u}{\partial x_{1}}\right)}$ \\
\hline $2^{-6}$ & $\left(2^{-4}, 2^{-13}\right)$ & 0.03 & 197.34 & $9.3475 \times 10^{-6}$ & 3.1457 \\
$2^{-5}$ & $\left(2^{-5}, 2^{-14}\right)$ & 0.09 & 1187.55 & $2.97147 \times 10^{-6}$ & 3.5508 \\
$2^{-4}$ & $\left(2^{-6}, 2^{-15}\right)$ & 0.59 & $18,501.80$ & $8.36840 \times 10^{-7}$ & 3.7737 \\
$2^{-3}$ & $\left(2^{-7}, 2^{-16}\right)$ & 3.69 & $144,505.21$ & $2.21757 \times 10^{-7}$ & \\
\hline
\end{tabular}

Table 2. Computational time, maximum norm of the errors, and the order of convergence of $z_{h, \tau}$ to the exact derivative $z=\frac{\partial u}{\partial x_{2}}$ when $r=\frac{0.5 \tau}{h^{2}} \leq \frac{3}{7}$.

\begin{tabular}{cccccc}
\hline$r=\frac{0.5 \tau}{h^{2}}$ & $(h, \tau)$ & $C T^{H^{2 n d}\left(\frac{\partial u}{\partial x_{2}}\right)}$ & $\boldsymbol{T C T} \boldsymbol{H}^{2 n d}\left(\frac{\partial u}{\partial x_{2}}\right)$ & $\left\|\varepsilon^{H^{2 n d}}\left(\frac{\partial u}{\partial x_{2}}\right)\right\|_{\infty}$ & $\Re^{H^{2 n d}\left(\frac{\partial u}{\partial x_{2}}\right)}$ \\
\hline $2^{-6}$ & $\left(2^{-4}, 2^{-13}\right)$ & 0.02 & 181.88 & $3.72134 \times 10^{-6}$ & 1.7362 \\
$2^{-5}$ & $\left(2^{-5}, 2^{-14}\right)$ & 0.13 & 1187.55 & $2.14336 \times 10^{-6}$ & 2.6720 \\
$2^{-4}$ & $\left(2^{-6}, 2^{-15}\right)$ & 0.70 & $21,557.80$ & $8.02154 \times 10^{-7}$ & 3.2757 \\
$2^{-3}$ & $\left(2^{-7}, 2^{-16}\right)$ & 4.09 & $169,305.04$ & $2.44880 \times 10^{-7}$ & \\
\hline
\end{tabular}

Table 3 presents the $C T^{H^{2 n d}\left(\frac{\partial u}{\partial x_{1}}\right)}, T C T^{H^{2 n d}\left(\frac{\partial u}{\partial x_{1}}\right)}$, maximum norm of the errors for $h=2^{-\mu}, \mu=4,5,6,7,8$ when $\tau=2^{-\lambda}, \lambda=8,9,10,11,12$, that is $r=\frac{0.5 \tau}{h^{2}}>\frac{3}{7}$ and the order of convergence of $v_{h, \tau}$ to the exact derivative $v=\frac{\partial u}{\partial x_{1}}$ with respect to $h$ and $\tau$ obtained by using the constructed two stage implicit method $H^{2 n d}\left(\frac{\partial u}{\partial x_{1}}\right)$. Table 4 shows the $C T^{H^{2 n d}}\left(\frac{\partial u}{\partial x_{2}}\right)$, $T C T^{H^{2 n d}\left(\frac{\partial u}{\partial x_{2}}\right)}$, maximum norm of the errors for the same pairs of $(h, \tau)$ as in Table 3 and the order of convergence of $z_{h, \tau}$ to the exact derivative $z=\frac{\partial u}{\partial x_{2}}$ with respect to $h$ and $\tau$ 
obtained by using the constructed two stage implicit method $H^{2 n d}\left(\frac{\partial u}{\partial x_{2}}\right)$. Numerical results given in Tables 3 and 4 demonstrate that when $r>\frac{3}{7}$, the approximate solutions $v_{h, \tau}$ and $z_{h, \tau}$ of the proposed method also converge with second order both in the spatial variables $x_{1}, x_{2}$ and the time variable $t$ to their corresponding exact derivatives $v=\frac{\partial u}{\partial x_{1}}$ and $z=\frac{\partial u}{\partial x_{2}}$.

Table 3. Computational time, maximum norm of the errors, and the order of convergence of $v_{h, \tau}$ to the exact derivative $v=\frac{\partial u}{\partial x_{1}}$ when $r=\frac{0.5 \tau}{h^{2}}>\frac{3}{7}$.

\begin{tabular}{cccccc}
\hline$r=\frac{0.5 \tau}{h^{2}}$ & $(h, \tau)$ & $C T^{H^{2 n d}\left(\frac{\partial u}{\partial x_{1}}\right)}$ & $T C T^{H^{2 n d}\left(\frac{\partial u}{\partial x_{1}}\right)}$ & $\left\|\varepsilon^{H^{2 n d}\left(\frac{\partial u}{\partial x_{1}}\right)}\right\|_{\infty}$ & $\Re^{H^{2 n d}\left(\frac{\partial u}{\partial x_{1}}\right)}$ \\
\hline $2^{-1}$ & $\left(2^{-4}, 2^{-8}\right)$ & 0.02 & 4.75 & $9.34796 \times 10^{-6}$ & 3.1458 \\
1 & $\left(2^{-5}, 2^{-9}\right)$ & 0.08 & 37.30 & $2.97159 \times 10^{-6}$ & 3.5508 \\
2 & $\left(2^{-6}, 2^{-10}\right)$ & 0.42 & 347.70 & $8.36871 \times 10^{-7}$ & 3.7737 \\
$2^{2}$ & $\left(2^{-7}, 2^{-11}\right)$ & 3.47 & 3988.83 & $2.21765 \times 10^{-7}$ & 3.8889 \\
$2^{3}$ & $\left(2^{-8}, 2^{-12}\right)$ & 41.25 & $68,313.10$ & $5.70258 \times 10^{-8}$ & \\
\hline
\end{tabular}

Table 4. Computational time, maximum norm of the errors, and the order of convergence of $z_{h, \tau}$ to the exact derivative $z=\frac{\partial u}{\partial x_{2}}$ when $r=\frac{0.5 \tau}{h^{2}}>\frac{3}{7}$.

\begin{tabular}{cccccc}
\hline$r=\frac{0.5 \tau}{h^{2}}$ & $(h, \tau)$ & $C T^{H^{2 n d}\left(\frac{\partial u}{\partial x_{2}}\right)}$ & $\boldsymbol{T C T} \boldsymbol{T}^{H^{2 n d}\left(\frac{\partial u}{\partial x_{2}}\right)}$ & $\| \varepsilon^{H^{2 n d}\left(\frac{\partial u}{\partial x_{2}}\right) \|_{\infty}}$ & $\Re^{H^{2 n d}\left(\frac{\partial u}{\partial x_{2}}\right)}$ \\
\hline $2^{-1}$ & $\left(2^{-4}, 2^{-8}\right)$ & 0.03 & 7.52 & $3.72102 \times 10^{-6}$ & 1.7361 \\
1 & $\left(2^{-5}, 2^{-9}\right)$ & 0.13 & 64.38 & $2.14327 \times 10^{-6}$ & 2.6720 \\
2 & $\left(2^{-6}, 2^{-10}\right)$ & 0.59 & 533.53 & $8.02135 \times 10^{-7}$ & 3.2757 \\
$2^{2}$ & $\left(2^{-7}, 2^{-11}\right)$ & 3.83 & 5122.09 & $2.44877 \times 10^{-7}$ & 3.6202 \\
$2^{3}$ & $\left(2^{-8}, 2^{-12}\right)$ & 42.91 & $73,957.51$ & $6.76426 \times 10^{-8}$ & \\
\hline
\end{tabular}

Figure 3 illustrates the absolute error functions $\left|\mathcal{E}^{H^{2 n d}\left(\frac{\partial u}{\partial x_{1}}\right)\left(2^{-4}, 2^{-13}\right)}\right|,\left|\varepsilon^{H^{2 n d}\left(\frac{\partial u}{\partial x_{1}}\right)\left(2^{-5}, 2^{-14}\right)}\right|$, $\left|\mathcal{E}^{H^{2 n d}\left(\frac{\partial u}{\partial x_{1}}\right)\left(2^{-6}, 2^{-15}\right)}\right|$, and $\left|\varepsilon^{H^{2 n d}\left(\frac{\partial u}{\partial x_{1}}\right)\left(2^{-7}, 2^{-16}\right)}\right|$ at time moment $t=0.2$ obtained by using $H^{2 n d}\left(\frac{\partial u}{\partial x_{1}}\right)$. Figure 4 demonstrates the absolute error functions $\left|\varepsilon^{H^{2 n d}}\left(\frac{\partial u}{\partial x_{2}}\right)\left(2^{-4}, 2^{-13}\right)\right|$, $\left|\varepsilon^{H^{2 n d}}\left(\frac{\partial u}{\partial x_{2}}\right)\left(2^{-5}, 2^{-14}\right)\right|,\left|\varepsilon^{H^{2 n d}\left(\frac{\partial u}{\partial x_{2}}\right)\left(2^{-6}, 2^{-15}\right)}\right|$, and $\left|\varepsilon^{H^{2 n d}\left(\frac{\partial u}{\partial x_{2}}\right)\left(2^{-7}, 2^{-16}\right)}\right|$ at time moment $t=0.2$ obtained by using $H^{2 n d}\left(\frac{\partial u}{\partial x_{2}}\right)$. The exact derivative $v=\frac{\partial u}{\partial x_{1}}$ and the grid function $v_{2^{-6}, 2^{-15}}$ for $h=2^{-6}, \tau=2^{-15}$ at time moment $t=0.2$ obtained by using $H^{2 n d}\left(\frac{\partial u}{\partial x_{1}}\right)$ are presented in Figure 5. Further, Figure 6 shows the exact derivative $z=\frac{\partial u}{\partial x_{2}}$ and grid function $z_{2^{-6}, 2^{-15}}$ at time moment $t=0.2$ obtained by using $H^{2 n d}\left(\frac{\partial u}{\partial x_{2}}\right)$. 

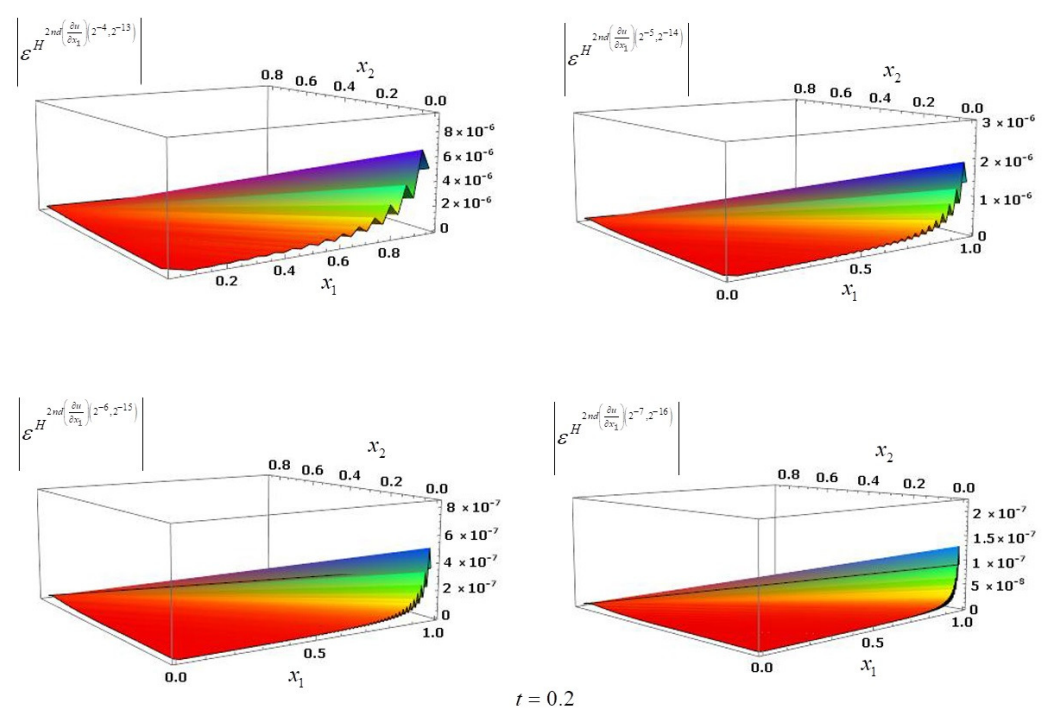

Figure 3. The absolute error functions at time moment $t=0.2$ obtained by using $H^{2 n d}\left(\frac{\partial u}{\partial x_{1}}\right)$.
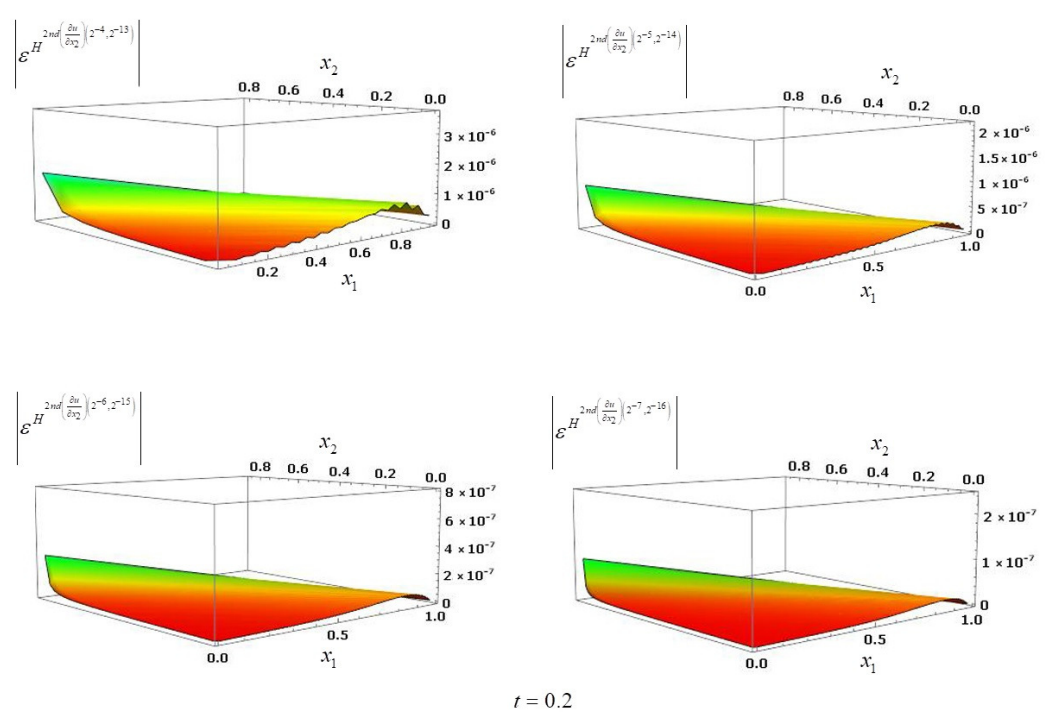

Figure 4. The absolute error functions at time moment $t=0.2$ obtained by using $H^{2 n d}\left(\frac{\partial u}{\partial x_{2}}\right)$.
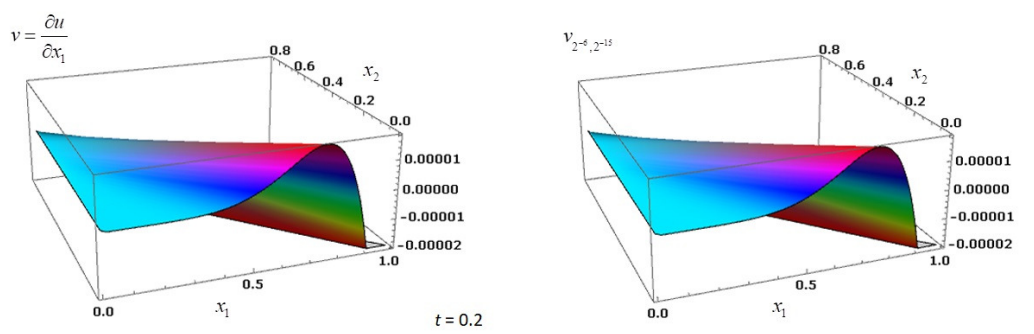

Figure 5. The exact solution $v=\frac{\partial u}{\partial x_{1}}$ and the approximate solution $v_{2^{-6}, 2^{-15}}$ at $t=0.2$. 

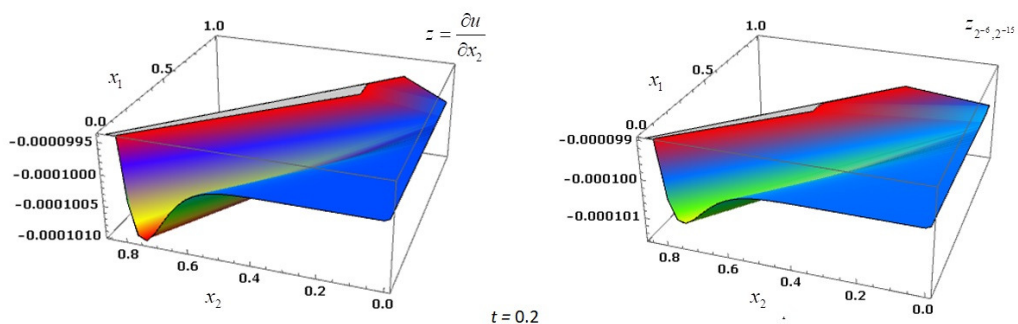

Figure 6. The exact solution $z=\frac{\partial u}{\partial x_{2}}$ and the approximate solution $z_{2^{-6}, 2^{-15}}$ at $t=0.2$.

Table 5 shows the $C T^{H^{2 n d}\left(\frac{\partial u}{\partial x_{1}}\right)}, T C T^{H^{2 n d}\left(\frac{\partial u}{\partial x_{1}}\right)}$, maximum norm of the errors for $r \leq \frac{3}{7}$, and the order of convergence of $v_{h, \tau}$ to the exact derivative $v=\frac{\partial u}{\partial x_{1}}$ with respect to $h$ and $\tau$ obtained when third order approximations for $v=\frac{\partial u}{\partial x_{1}}$

$$
\begin{gathered}
p_{1 h}^{3^{r d}}=\left\{\begin{array}{c}
\frac{1}{6 h}\left(-11 u\left(0, x_{2}, t\right)+18 u_{h, \tau}\left(h, x_{2}, t\right)\right. \\
\left.-9 u_{h, \tau}\left(2 h, x_{2}, t\right)+2 u_{h, \tau}\left(3 h, x_{2}, t\right)\right) \text { if } P_{0} \in D^{0 h} \gamma_{\tau} \\
\frac{1}{60 h}\left(-184 u\left(0, x_{2}, t\right)+225 u_{h, \tau}\left(\frac{h}{2}, x_{2}, t\right)\right. \\
\left.-50 u_{h, \tau}\left(\frac{3 h}{2}, x_{2}, t\right)+9 u_{h, \tau}\left(\frac{5 h}{2}, x_{2}, t\right)\right) \text { if } P_{0} \in D^{* l h} \gamma_{\tau}
\end{array} \text { on } S_{T}^{h} \gamma_{1},\right. \\
p_{3 h}^{3^{r d}}=\left\{\begin{array}{c}
\frac{1}{6 h}\left(11 u\left(a_{1}, x_{2}, t\right)-18 u_{h, \tau}\left(a_{1}-h, x_{2}, t\right)\right. \\
\left.+9 u_{h, \tau}\left(a_{1}-2 h, x_{2}, t\right)-2 u_{h, \tau}\left(a_{1}-3 h, x_{2}, t\right)\right) \text { if } P_{0} \in D^{0 h} \gamma_{\tau} \\
\frac{1}{60 h}\left(184 u\left(a_{1}, x_{2}, t\right)-225 u_{h, \tau}\left(a_{1}-\frac{h}{2}, x_{2}, t\right)\right. \\
\left.+50 u_{h, \tau}\left(a_{1}-\frac{3 h}{2}, x_{2}, t\right)-9 u_{h, \tau}\left(a_{1}-\frac{5 h}{2}, x_{2}, t\right)\right) \text { if } P_{0} \in D^{* r h} \gamma_{\tau}
\end{array} \text { on } S_{T}^{h} \gamma_{3},\right.
\end{gathered}
$$

are used on $S_{T}^{h} \gamma_{i}, i=1,3$ for the Stage $2\left(H^{2 n d}\left(\frac{\partial u}{\partial x_{1}}\right)\right)$. Table 6 shows $C T^{H^{2 n d}\left(\frac{\partial u}{\partial x_{2}}\right)}$, $T C T^{H^{2 n d}\left(\frac{\partial u}{\partial x_{2}}\right)}$, maximum norm of the errors for $r \leq \frac{3}{7}$ and the order of convergence of $z_{h, \tau}$ to the exact derivative $z=\frac{\partial u}{\partial x_{2}}$ with respect to $h$ and $\tau$ obtained when third order approximations for $z=\frac{\partial u}{\partial x_{2}}$.

$$
\begin{aligned}
q_{2 h}^{3 r d}= & \frac{1}{6 \sqrt{3} h}\left(-11 u\left(x_{1}, 0, t\right)+18 u_{h, \tau}\left(x_{1}, \sqrt{3} h, t\right)\right. \\
& \left.-9 u_{h, \tau}\left(x_{1}, 2 \sqrt{3} h, t\right)+2 u_{h, \tau}\left(x_{1}, 3 \sqrt{3} h, t\right)\right) \text { on } S_{T}^{h} \gamma_{2}, \\
q_{4 h}^{3^{r d}}= & \frac{1}{6 \sqrt{3} h}\left(11 u\left(x_{1}, a_{2}, t\right)-18 u_{h, \tau}\left(x_{1}, a_{2}-\sqrt{3} h, t\right)\right. \\
& \left.+9 u_{h, \tau}\left(x_{1}, a_{2}-2 \sqrt{3} h, t\right)-2 u_{h, \tau}\left(x_{1}, a_{2}-3 \sqrt{3} h, t\right)\right) \text { on } S_{T}^{h} \gamma_{4},
\end{aligned}
$$

are used on $S_{T}^{h} \gamma_{i}, i=2,4$ for the Stage $2\left(H^{2 n d}\left(\frac{\partial u}{\partial x_{2}}\right)\right)$. Table 7 presents $C T^{H^{2 n d}}\left(\frac{\partial u}{\partial x_{1}}\right)$, $T C T^{H^{2 n d}\left(\frac{\partial u}{\partial x_{1}}\right)}$, maximum norm of the errors for $r>\frac{3}{7}$, and the order of convergence of $v_{h, \tau}$ to the exact derivatives $v=\frac{\partial u}{\partial x_{1}}$ with respect to $h$ and $\tau$ obtained by using the difference formulae (172), (173) on $S_{T}^{h} \gamma_{i}, i=1,3$ for the Stage $2\left(H^{2 n d}\left(\frac{\partial u}{\partial x_{1}}\right)\right)$. Table 8 gives $C T^{H^{2 n d}\left(\frac{\partial u}{\partial x_{2}}\right)}, T C T^{H^{2 n d}\left(\frac{\partial u}{\partial x_{2}}\right)}$, maximum norm of the errors for $r>\frac{3}{7}$, and the order of convergence of $z_{h, \tau}$ to the exact derivative $z=\frac{\partial u}{\partial x_{2}}$ with respect to $h$ and $\tau$ obtained by using the difference formulae (174), (175) on $S_{T}^{h} \gamma_{i}, i=2,4$ for the Stage $2\left(H^{2 n d}\left(\frac{\partial u}{\partial x_{2}}\right)\right)$. Numerical results given in Tables 5-8 demonstrate that the approximate solution $v_{h, \tau}$ and $z_{h, \tau}$ of the proposed method converges to the corresponding exact derivatives $v=\frac{\partial u}{\partial x_{1}}$ and 
$z=\frac{\partial u}{\partial x_{2}}$ with second order both in the spatial variables $x_{1}, x_{2}$ and the time variable $t$ with better error ratios.

Table 5. Computational time, maximum norm of the errors, and the order of convergence of $v_{h, \tau}$ to the exact derivative $v=\frac{\partial u}{\partial x_{1}}$ when (172) and (173) are used and $r=\frac{0.5 \tau}{h^{2}} \leq \frac{3}{7}$.

\begin{tabular}{cccccc}
\hline $\boldsymbol{r}=\frac{\mathbf{0 . 5 \tau}}{\boldsymbol{h}^{\mathbf{2}}}$ & $(\boldsymbol{h}, \boldsymbol{\tau})$ & $\boldsymbol{C} \boldsymbol{T}^{\boldsymbol{H}^{2 n d}\left(\frac{\partial u}{\partial x_{1}}\right)}$ & $\boldsymbol{T C T ^ { \boldsymbol { H } ^ { 2 n d } } ( \frac { \partial u } { \partial x _ { 1 } } )}$ & $\left\|\varepsilon^{H^{2 n d}\left(\frac{\partial u}{\partial x_{1}}\right)}\right\|_{\infty}$ & $\Re^{H^{2 n d}\left(\frac{\partial u}{\partial x_{1}}\right)}$ \\
\hline $2^{-6}$ & $\left(2^{-4}, 2^{-13}\right)$ & 0.03 & 216.25 & $3.93819 \times 10^{-6}$ & 4.5863 \\
$2^{-5}$ & $\left(2^{-5}, 2^{-14}\right)$ & 0.09 & 1695.39 & $8.58690 \times 10^{-7}$ & 4.6031 \\
$2^{-4}$ & $\left(2^{-6}, 2^{-15}\right)$ & 0.63 & 18945.40 & $1.86547 \times 10^{-7}$ & 4.6131 \\
$2^{-3}$ & $\left(2^{-7}, 2^{-16}\right)$ & 3.67 & 218517.01 & $4.04385 \times 10^{-8}$ & \\
\hline
\end{tabular}

Table 6. Computational time, maximum norm of the errors, and the order of convergence of $z_{h, \tau}$ to the exact derivative $z=\frac{\partial u}{\partial x_{2}}$ when (174) and (175) are used and $r=\frac{0.5 \tau}{h^{2}} \leq \frac{3}{7}$.

\begin{tabular}{|c|c|c|c|c|c|}
\hline$r=\frac{0.5 \tau}{h^{2}}$ & $(h, \tau)$ & $C T^{H^{2 n d}\left(\frac{\partial u}{\partial x_{2}}\right)}$ & $T C T^{H^{2 n d}\left(\frac{\partial u}{\partial x_{2}}\right)}$ & $\varepsilon^{H^{2 n d}\left(\frac{\partial u}{\partial x_{2}}\right)} \|_{\infty}$ & $\Re^{H^{2 n d}\left(\frac{\partial u}{\partial x_{2}}\right)}$ \\
\hline $2^{-6}$ & $\left(2^{-4}, 2^{-13}\right)$ & 0.03 & 251.27 & $3.37221 \times 10^{-6}$ & 2.5722 \\
\hline $2^{-5}$ & $\left(2^{-5}, 2^{-14}\right)$ & 0.13 & 2088.16 & $1.31103 \times 10^{-6}$ & 4.3277 \\
\hline $2^{-4}$ & $\left(2^{-6}, 2^{-15}\right)$ & 0.63 & 18945.40 & $3.02939 \times 10^{-7}$ & 4.4163 \\
\hline $2^{-3}$ & $\left(2^{-7}, 2^{-16}\right)$ & 3.85 & 234313.60 & $6.85956 \times 10^{-8}$ & \\
\hline
\end{tabular}

Table 7. Computational time, maximum norm of the errors, and the order of convergence of $v_{h, \tau}$ to the exact derivative $v=\frac{\partial u}{\partial x_{1}}$ when (172) and (173) are used and $r=\frac{0.5 \tau}{h^{2}}>\frac{3}{7}$.

\begin{tabular}{cccccc}
\hline$r=\frac{\mathbf{0 . 5 \tau}}{\boldsymbol{h}^{\mathbf{2}}}$ & $(h, \tau)$ & $\boldsymbol{C} T^{H^{2 n d}\left(\frac{\partial u}{\partial x_{1}}\right)}$ & $\boldsymbol{T C T ^ { H ^ { 2 n d } } ( \frac { \partial u } { \partial x _ { 1 } } )}$ & $\left\|\varepsilon^{H^{2 n d}\left(\frac{\partial u}{\partial x_{1}}\right)}\right\|_{\infty}$ & $\Re^{H^{2 n d}\left(\frac{\partial u}{\partial x_{1}}\right)}$ \\
\hline $2^{-1}$ & $\left(2^{-4}, 2^{-8}\right)$ & 0.02 & 5.08 & $3.93866 \times 10^{-6}$ & 4.5862 \\
1 & $\left(2^{-5}, 2^{-9}\right)$ & 0.08 & 38.19 & $8.58815 \times 10^{-7}$ & 4.6030 \\
2 & $\left(2^{-6}, 2^{-10}\right)$ & 0.44 & 352.03 & $1.86579 \times 10^{-7}$ & 4.4176 \\
$2^{2}$ & $\left(2^{-7}, 2^{-11}\right)$ & 3.52 & 3994.16 & $4.22355 \times 10^{-8}$ & \\
\hline
\end{tabular}

Table 8. Computational time, maximum norm of the errors, and the order of convergence of $z_{h, \tau}$ to the exact derivative $z=\frac{\partial u}{\partial x_{2}}$ when (174) and (175) are used and $r=\frac{0.5 \tau}{h^{2}}>\frac{3}{7}$.

\begin{tabular}{|c|c|c|c|c|c|}
\hline$r=\frac{0.5 \tau}{h^{2}}$ & $(h, \tau)$ & $C T^{H^{2 n d}\left(\frac{\partial u}{\partial x_{2}}\right)}$ & $T C T^{H^{2 n d}}\left(\frac{\partial u}{\partial x_{2}}\right)$ & $\left|{ }_{\mathcal{E}} H^{2 n d}\left(\frac{\partial u}{\partial x_{2}}\right)\right| \mid$ & $\Re^{H^{2 n d}}\left(\frac{\partial u}{\partial x_{2}}\right)$ \\
\hline $2^{-1}$ & $\left(2^{-4}, 2^{-8}\right)$ & 0.02 & 5.89 & $3.67669 \times 10^{-6}$ & 2.8278 \\
\hline 1 & $\left(2^{-5}, 2^{-9}\right)$ & 0.11 & 45.67 & $1.30019 \times 10^{-6}$ & 4.4268 \\
\hline 2 & $\left(2^{-6}, 2^{-10}\right)$ & 0.50 & 414.27 & $2.93712 \times 10^{-7}$ & 4.5165 \\
\hline $2^{2}$ & $\left(2^{-7}, 2^{-11}\right)$ & 3.72 & 4475.91 & $6.50300 \times 10^{-8}$ & \\
\hline
\end{tabular}

\section{Discussions and Conclusion}

A second order accurate two stage implicit method on hexagonal grids is proposed for the approximation of the first order derivatives of the solution to first type boundary value problem of the heat equation with respect to spatial variables on rectangle. At the first stage, for the error function, we provided a pointwise prior estimation depending on $\rho\left(x_{1}, x_{2}, t\right)$, which is the distance from the current grid point in the domain to the surface of $Q_{T}$. At the second stage, we constructed special difference problems for the approximation of $\frac{\partial u}{\partial x_{1}}$ and $\frac{\partial u}{\partial x_{2}}$. Uniform convergence with order $O\left(h^{2}+\tau^{2}\right)$ of the solution of the constructed difference problems to the corresponding exact derivatives $\frac{\partial u}{\partial x_{1}}$ and $\frac{\partial u}{\partial x_{2}}$ on the hexagonal grids when $r=\frac{\omega \tau}{h^{2}} \leq \frac{3}{7}$ is proved. Furthermore, the given two stage implicit method 
is applied on a test problem and the obtained theoretical order of convergence is shown numerically using tables and figures.

Remark 1. The methodology given in this research may be used to construct highly accurate implicit splitting schemes (fractional step methods) and alternating direction methods (ADI) (see [40-43]) for the approximation of the first order partial derivatives of solution of first type boundary value problem of heat equation in three space dimension. Additionally, this approach may be extended to find the spatial derivatives of the solution of the time-fractional structure of the heat equation. Since as well as its solution, the computation of the derivatives of the solution of the fractional model problem are essential. Such as the time-space fractional convection-diffusion equation, see [44], in which for the solution a fast iterative method with a second-order implicit difference scheme was studied.

Author Contributions: Conceptualization, S.C.B.; methodology, S.C.B.; software, N.A. and A.H.M.; validation, S.C.B., A.H.M., and N.A.; formal analysis, S.C.B., A.H.M., and N.A; investigation, A.H.M. and N.A.; resources, S.C.B., A.H.M., and N.A.; writing-review and editing, S.C.B., A.H.M., and N.A.; visualization, S.C.B., A.H.M., and N.A.; supervision, S.C.B.; project administration, S.C.B. All authors have read and approved the final manuscript.

Funding: This research did not receive any specific grant from funding agencies in the public, commercial, or not-for-profit sectors.

Informed Consent Statement: Not applicable.

Data Availability Statement: Not applicable.

Conflicts of Interest: The authors declare no conflict of interest.

\section{References}

1. Doungmo Goufo, E.F. Evolution equations with a parameter and application to transport-convection differential equations. Turk. J. Math. 2017, 41, 636-654. [CrossRef]

2. Doungmo Goufo, E.F. Solvability of chaotic fractional systems with 3D four-scroll attractors. Chaos Solitons Fractals 2017, 104, 443-451. [CrossRef]

3. Doungmo Goufo, E.F. The Proto-Lorenz system in its chaotic fractional and fractal structure. Int. J. Bifurcation Chaos 2020, 30, 2050180. [CrossRef]

4. Doungmo Goufo, E.F.; Khan, Y. A new auto-replication in systems of attractors with two and three merged basins of attraction via control. Commun. Nonlinear Sci. Numer. Simulat. 2021, 96, 105709. [CrossRef]

5. Owolabi, K.M.; Hammouch, Z. Mathematical modeling and analysis of two-variable system with noninteger-order derivative. Chaos 2019, 29, 013145. [CrossRef] [PubMed]

6. Doungmo Goufo, E.F.; Khan, Y.; Mugisha, S. Control parameter \& solutions to generalized evolution equations of stationarity, relaxation and diffusion. Results Phys. 2018, 9, 1502-1507.

7. Doungmo Goufo, E.F.; Kumar, S.; Mugisha, S.B. Similarities in a fifth-order evolution equation with and with no singular kernel. Chaos Solitons Fractals 2020, 130, 109467. [CrossRef]

8. Heydari, M.H.; Hooshmandasl, M.R.; Mohammadi, F.; Cattani, C. Wavelets method for solving systems of nonlinear singular fractional Volterra integro-differential equations. Commun. Nonlinear Sci. Numer. Simulat. 2014, 19, 37-48. [CrossRef]

9. Čermák, J.; Kisela, T. Delay-dependent stability switches in fractional differential equations. Commun. Nonlinear Sci. Numer. Simulat. 2019, 79, 104888. [CrossRef]

10. Tuladhar, R.; Santamaria, F.; Stamova, I. Fractional Lotka-Volterra-Type Cooperation Models: Impulsive control on their stability behavior. Entropy 2020, 22, 970; doi:10.3390/e22090970. [CrossRef]

11. Zhang, H.; Sandu, A. A second-order diagonally-implicit-explicit multi-stage integration method. Procedia Computer Sci. 2012, 9, 1039-1046. [CrossRef]

12. Hasan, M.K.; Ahamed, M.S.; Alam, M.S.; Hossain, M.B. An implicit method for numerical solution of singular and stiff initial value problems. J. Comput. Eng. 2013, 2013, 1-5. [CrossRef]

13. Ashyralyev, A.; Hincal, E.; Kaymakamzade, B. A Crank Nicolson difference scheme for system of nonlinear observing epidemic models. In AIP Conference Proceedings; AIP Publishing LLC: Melville, NY, USA, 2019; Volume 2183.

14. Ahmed, N.; Ali, M.; Baleanu, D.; Rafiq, M.; ur Rehman, M.A. Numerical analysis of diffusive susceptible-infected-recovered epidemic model in three space dimension. Chaos Solitons Fractals 2020, 132, 1-10. [CrossRef]

15. Sadourney, R.; Arakawa, A.; Mintz,Y. Integration of the nondivergent barotropic vorticity equation with an icosahedral-hexagonal grid for the sphere. Mon. Wea. Rev. 1968, 96, 351-356. [CrossRef] 
16. Williamson, D. Integration of the barotropic vorticity equation on a spherical geodesic grid. Tellus 1968, 20, 642-653. [CrossRef]

17. Sadourney, R. Numerical integration of the primitive equation on a spherical grid with hexagonal cells. In Proceedings of the WMO/IUGG Symposium on Numerical Whether Prediction, Tokyo, Japan, 26 November-4 December 1969; pp. 45-77.

18. Sadourney, R.; Morel, P. A finite-difference approximation of the primitive equations for a hexagonal grid on a plane. Mon. Wea. Rev. 1969, 97, 439-445. [CrossRef]

19. Masuda, Y. A finite difference scheme by making use of hexagonal mesh-points. In Proceedings of the WMO/IUGG Symposium on Numerical Whether Prediction, Tokyo, Japan, 26 November-4 December 1969; pp. VII35-VII44.

20. Masuda, Y.; Ohnishi H. An integration scheme of the primitive equation model with an icosahedral-hexagonal grid system and its application to the shallow water equation. J. Meteorol. Soc. Jpn. 1986, 64, 317-326. [CrossRef]

21. Thacker, W.C. Irregular grid finite difference techniques: Simulations of oscillations in shallow circular basins. J. Phys. Oceanogr. 1977, 7, 284-292. [CrossRef]

22. Thacker, W.C. Comparison of finite element and finite difference schemes, Part II: Two dimensional gravity wave motion. J. Phys. Oceanogr. 1978, 8, 680-689. [CrossRef]

23. Salmon, R.; Talley, D.L. Generalization of Arakawa's Jacobian. J. Comput. Physics 1989, 83, 247-259. [CrossRef]

24. Ničkovič, S. On the use of hexagonal grids for simulation of atmospheric processes. Contrib. Atmos. Phys. 1994, 67, $103-107$.

25. Ničkovič, S.; Gavrilov, M.B.; Tosič, I.A. Geostrophic adjuctment on hexagonal grids. Mon. Wea. Rev. 2001, 130, 668-683. [CrossRef]

26. Pruess, K.; Bodvarsson, G.S. A Seven-Point Finite Difference method For Improved Grid Orientation Performance in Pattern Steam Floods; Lawrence Berkerly National Laboratory: Berkeley, CA, USA, 1983; pp. 1-32.

27. Lee, D.; Tien, H.-C.; Luo, C.P.; Luk, H.-N. Hexagonal grid methods with applications to partial differential equations. Int. J. Comput. Math. 2014, 91, 1986-2009. [CrossRef]

28. Richtmyer, R.D.; Morton, K.W. Difference Methods for Initial-Value Problems, 2nd ed.; Interscience Publishers a Division of Jhon Wiley and Sons: Hoboken, NJ, USA, 1967.

29. Buranay, S.C.; Arshad, N. Hexagonal grid approximation of the solution of heat equation on special polygons. Adv. Differ. 2020, 2020, 309. [CrossRef]

30. Arshad, N. Hexagonal Grid Approximation of the Solution of Two Dimensional Heat Equation. Ph.D. Thesis, Eastern Mediterranean University, Famagusta, Cyprus, 2020.

31. Karaa, S. High-order approximation of 2D convection-diffusion equation on hexagonal grids. Numer. Methods Partial. Differ. Equations 2006, 22, 1238-1246. [CrossRef]

32. Dosiyev, A.A.; Celiker, E. Approximation on the hexagonal grid of the Dirichlet problem for Laplace's equation. Boundary Value Probl. 2014, 73, 1-19. [CrossRef]

33. Lax, P.D.; Richtmyer, R.D. Survey of the stability of linear finite difference equations. Commun. Pure Applied Mathematics 1956, 9, 267-293. [CrossRef]

34. Buranay, S.C.; Iyikal, O.C. Incomplete block-matrix factorization of $M$-matrices using two step iterative method for matrix inversion and preconditioning. Math. Methods Appl. Sci. 2020, in press. [CrossRef]

35. Concus, P.; Golub, G.H.; Meurant, G. Block preconditioning for the conjugate gradient method. SIAM J. 1985, 6, 220-252. [CrossRef]

36. Axelsson, O. A general incomplete block matrix factorization method. Linear Algebra Its Appl. 1986, 74, 179-190. [CrossRef]

37. Azzam, A.; Kreyszig, E. On solutions of parabolic equations in regions with edges. Bull. Austral. Math. Soc. 1980, 22, 219-230. [CrossRef]

38. Azzam, A.; Kreyszig, E. Smoothness of solutions of parabolic equations in regions with edges. Nagoya Math. J. 1981, 84, 159-168. [CrossRef]

39. Burden, R.L.; Faires, J.D. Numerical Analysis, 9th ed.; Cengage Learning: Boston, MA, USA, 2011.

40. Peaceman, D.W.; Rachford, H.H., Jr. The numerical solution of parabolic and elliptic differential equations. J. Soc. Industrial Appl. Math. 1955, 3, 28-41. [CrossRef]

41. Douglas, J. On the numerical integration of $\frac{\partial^{2} u}{\partial x^{2}}+\frac{\partial^{2} u}{\partial y^{2}}=\frac{\partial u}{\partial t}$ by implicit methods. J. Soc. Industrial Appl. Math. 1955, 3, 42-65.

42. Bagrinovskii, K.A.; Godunov, S.K. Difference schemes for multidimensional problems. Dokl. Akad. Nauk USSR 1957, 115, 431-433.

43. Marchuk, G.I. Splitting and Alternating Methods. In Handbook of Numerical Analysis; Elsevier Science Publishers: Amsterdam, The Netherlands, 1990.

44. Gu, X.M.; Huang, T.Z.; Ji, C.C.; Carpentieri, B.; Alikhanov, A.A. Fast iterative method with a second-order implicit difference scheme for time-space fractional convection-diffusion equation. J. Sci. Comput. 2017, 72, 957-985. [CrossRef] 